\title{
Improved Basis-Set Incompleteness Potentials for Accurate DFT Calculations in Large Systems
}

\author{
A. Otero-de-la-Roza* \\ Departamento de Química Física y Analítica and MALTA-Consolider Team, \\ Facultad de Química, Universidad de Oviedo, 33006 Oviedo, Spain \\ Gino A. DiLabio ${ }^{\dagger}$ \\ Department of Chemistry, University of British Columbia, Okanagan, \\ 3247 University Way, Kelowna, British Columbia, Canada V1V $1 \mathrm{~V} \%$. and \\ Faculty of Management, University of British Columbia, Okanagan, \\ 1137 Alumni Avenue, Kelowna, British Columbia, V1V 1V7, Canada.
}

(Dated: October 22, 2019)

TABLE I. Mean absolute errors (MAE) for five basis sets (MINI, MINIs, STO-3G, 3-21G, and 6-31G) in the individual subsets of the training set using B3LYP against the near-complete-basis-set reference (B3LYP/aug-cc-pVQZ). Bare = the uncorrected basis set, LS = the least-squares fit prediction of the BSIP-corrected result, Eval = the actual BSIP result when used in a self-consistent calculation.

\begin{tabular}{|c|c|c|c|c|c|c|c|c|c|c|c|c|c|c|c|}
\hline & \multicolumn{3}{|c|}{ MINI } & \multicolumn{3}{|c|}{ MINIs } & \multicolumn{3}{|c|}{ STO-3G } & \multicolumn{3}{|c|}{$3-21 \mathrm{G}$} & \multicolumn{3}{|c|}{$6-31 \mathrm{G}$} \\
\hline Set & Bare & LS & Eval & Bare & LS & Eval & Bare & LS & Eval & Bare & LS & Eval & Bare & LS & Eval \\
\hline $\mathrm{S} 22 \times 5$ & 3.56 & 0.50 & 0.52 & 1.85 & 0.41 & 0.35 & 2.47 & 0.61 & 0.53 & 3.82 & 0.43 & 0.73 & 1.85 & 0.32 & 0.47 \\
\hline $\mathrm{S} 66 \times 8$ & 2.91 & 0.54 & 0.52 & 1.36 & 0.39 & 0.32 & 1.56 & 0.50 & 0.44 & 3.57 & 0.46 & 0.65 & 1.81 & 0.29 & 0.38 \\
\hline F-set & 3.53 & 0.95 & 4 & 2.02 & 0.64 & 9 & 93 & 0.77 & 11 & 3.05 & 0.73 & 0.89 & 1.89 & 0.39 & 0.51 \\
\hline P-set & 3.51 & 0.89 & 0.95 & 1.80 & 0.51 & 0.43 & 2.07 & 0.81 & 0.90 & 2.86 & 0.78 & 0.93 & 1.51 & 0.40 & 0.54 \\
\hline S-set & 1.79 & 0.60 & 0.60 & 0.67 & 0.45 & 0.39 & 0.85 & 0.48 & 0.47 & 2.29 & 0.42 & 0.42 & 1.12 & 0.28 & 0.27 \\
\hline Cl-set & 3.81 & 0.55 & 0.60 & 2.18 & 0.50 & 0.44 & 2.04 & 0.63 & 0.77 & 2.58 & 0.59 & 0.53 & 1.40 & 0.33 & 0.33 \\
\hline B-set & 95 & 1.18 & 1.40 & 50 & 0.88 & 1.06 & 5.86 & 0.84 & 1.24 & 5.36 & 0.67 & 04 & 2.64 & 0.48 & 0.73 \\
\hline Si-set & 3.70 & 1.02 & 1.15 & 1.85 & 0.63 & 0.73 & 2.03 & 0.91 & 1.02 & 3.61 & 0.81 & 1.06 & 1.82 & 0.65 & 0.90 \\
\hline KB49 & 3.70 & 0.81 & 0.87 & 1.84 & 0.59 & 0.55 & 2.54 & 0.85 & 0.93 & 4.24 & 0.53 & 0.90 & 2.05 & 0.38 & 0.56 \\
\hline Water & 95.43 & 1.13 & 7.57 & 56.39 & 1.04 & 4.38 & 63.44 & 1.57 & 9.02 & 89.22 & 1.67 & 16.20 & 48.88 & 0.73 & 10.11 \\
\hline A24 & 98 & 0.58 & 0 & 93 & 0.56 & 2 & 1.09 & 0.69 & 0.93 & 2.66 & 0.56 & 00 & 1.49 & 0.34 & 0.47 \\
\hline HSG & 85 & 68 & 0.90 & 73 & 0.51 & 0 & 2.26 & 0.70 & 0.73 & 4.13 & 0.35 & 80 & 2.01 & 0.23 & .48 \\
\hline IonicHB & 9.62 & 1.73 & 2.51 & 6.73 & 1.45 & 1.65 & 8.88 & 1.75 & 2.65 & 10.10 & 0.88 & 2.25 & 6.16 & 0.74 & 1.58 \\
\hline ADIM6 & 2.31 & 0.97 & 0.48 & 0.51 & 1.51 & 0.35 & 0.40 & 0.12 & 0.49 & 2.16 & 1.83 & 0.09 & 0.78 & 1.18 & 0.40 \\
\hline $0 x$ & 25 & .84 & & 14 & 0.78 & & 38 & 0.77 & 0.94 & 3.91 & 0.82 & 0.99 & 2.19 & 0.49 & 0.63 \\
\hline $\mathrm{S} \times 8$ & 1.67 & 0.50 & 0.50 & 75 & 0.35 & 0.33 & 0.89 & 0.49 & 0.50 & 2.67 & 0.39 & 0.45 & 1.38 & 0.29 & 0.29 \\
\hline BBI & 3.28 & 0.96 & 0.43 & 1.85 & 0.76 & 0.49 & 1.75 & 0.89 & 0.69 & 5.38 & 0.68 & 0.48 & 2.58 & 0.25 & 0.45 \\
\hline SSI & 3.11 & 0.54 & 0.72 & 1.40 & 0.46 & 0.47 & 2.21 & 0.58 & 0.78 & 3.07 & 0.35 & 0 & 1.50 & 0.25 & 0.39 \\
\hline $\mathrm{ACHC}$ & 5 & 0.53 & 0 & 4 & 0.53 & 0 . & 1.29 & 0.66 & 0.95 & 3.20 & 0.19 & 0.64 & 1.61 & 0.13 & 0.41 \\
\hline HBC6 & 9.26 & 0.68 & 0.97 & 6.94 & 0.66 & 0.65 & 9.55 & 0.91 & 0.87 & 9.71 & 0.81 & 2.04 & 4.56 & 0.58 & 1.18 \\
\hline NBC10ext & 2.11 & 0.44 & 0.51 & 0.89 & 0.37 & 0.39 & 1.20 & 0.52 & 0.55 & 1.43 & 0.23 & 0.28 & 0.80 & 0.25 & 0.26 \\
\hline Bauza & 6.96 & 14.70 & 14.71 & 5.80 & 15.52 & 15.29 & 5.31 & 11.18 & 10.20 & 5.62 & 7.71 & 6.77 & 4.35 & 7.48 & 6.50 \\
\hline P26 & 6.90 & 2.32 & 2. & 5.12 & 1.48 & 1 . & 5.47 & 1.70 & 1 . & 2.82 & 1.36 & 1.18 & 1.35 & 0.79 & 0.66 \\
\hline ACONF & 1.77 & 1.31 & 1.55 & 1.30 & 0.54 & 0.62 & 0.62 & 0.32 & 0.52 & 0.50 & 0.25 & 0.20 & 0.19 & 0.14 & 0.15 \\
\hline $\mathrm{CYCONF}$ & 4.14 & 2.93 & 3.36 & 3.19 & 1.23 & 1.38 & 3.30 & 0.97 & 1.07 & 2.80 & 0.67 & 0.63 & 1.23 & 0.38 & 0.30 \\
\hline SCONF & 11.34 & 0.97 & 0.87 & 9.52 & 1.12 & 1.51 & 9.20 & 1.04 & 1.52 & 9.63 & 0.77 & 2.01 & 3.94 & 0.63 & 1.47 \\
\hline BCONF & 46 & 0.90 & 1.26 & 4.62 & 0.51 & 0.43 & 5.56 & 0.93 & 0.71 & 5.38 & 0.77 & 0.66 & 2.84 & 0.53 & 0.38 \\
\hline BDE03 & 16.22 & 17.39 & 17.02 & 15.42 & 13.37 & 14.74 & 21.05 & 18.76 & 11.43 & 4.48 & 9.99 & 9.63 & 4.83 & 8.47 & 8.22 \\
\hline BHPeri & 28.69 & 8.13 & 7.08 & 13.38 & 4.86 & 5.15 & 15.11 & 4.53 & 5.68 & 9.28 & 7.12 & 8.25 & 3.61 & 3.93 & 3.91 \\
\hline BSR36 & 19.86 & 1.91 & 1.78 & 2.37 & 1.92 & 1.95 & 2.32 & 2.08 & 2.31 & 5.53 & 1.89 & 2.15 & 1.19 & 0.61 & 0.52 \\
\hline DARC & 66.99 & 21.10 & 9.15 & 9.36 & 15.40 & 19.38 & 42.09 & 4.05 & 13.33 & 17.10 & 16.34 & 17.97 & 4.73 & 6.15 & 4.10 \\
\hline BH76 & 19.25 & 14.33 & 13.81 & 19.09 & 12.17 & 11.82 & 21.63 & 15.85 & 15.62 & 11.22 & 7.59 & 8.78 & 7.17 & 6.71 & 6.93 \\
\hline ISO34 & 10.35 & 9.63 & 9.16 & 8.00 & 4.36 & 4.75 & 11.32 & 4.49 & 6.62 & 4.38 & 4.01 & 5.83 & 2.12 & 4.08 & 4.91 \\
\hline PA26 & 52.27 & 35.14 & 33.93 & 14.47 & 31.08 & 33.89 & 41.79 & 19.25 & 16.87 & 8.66 & 7.59 & 9.64 & 6.75 & 6.34 & 6.82 \\
\hline W4-11 & 16.801 & 124.641 & 119.82 & 55.55 & 86.14 & 81.79 & 42.941 & 107.36 & 67.68 & 29.07 & 39.01 & 39.93 & 35.44 & 34.59 & 37.07 \\
\hline BDExrel & 9.92 & 1.71 & 1.80 & 4.53 & 0.98 & 0.99 & 3.56 & 1.16 & 1.46 & 1.70 & 0.79 & 0.90 & 1.80 & 0.53 & 0.57 \\
\hline
\end{tabular}


TABLE II. Mean absolute errors (MAE) for five basis sets (MIDIh, pc-0, 6-31+G, 3-21G*, and 6-31G*) in the individual subsets of the training set using B3LYP against the near-complete-basis-set reference (B3LYP/aug-cc-pVQZ). Bare = the uncorrected basis set, LS = the least-squares fit prediction of the BSIP-corrected result, Eval = the actual BSIP result when used in a self-consistent calculation.

\begin{tabular}{|c|c|c|c|c|c|c|c|c|c|c|c|c|c|c|c|}
\hline \multirow[b]{2}{*}{ Set } & \multicolumn{3}{|c|}{ MIDIh } & \multicolumn{3}{|c|}{$\overline{\mathrm{pc}-0}$} & \multicolumn{3}{|c|}{$6-31+G$} & \multicolumn{3}{|c|}{$3-21 \mathrm{G}^{*}$} & \multicolumn{3}{|c|}{ 6-31G* } \\
\hline & Bare & LS & Eval & Bare & LS & Eval & Bare & LS & Eval & Bare & LS & Eval & Bare & LS & Eval \\
\hline $\mathrm{S} 22 \times 5$ & 1.85 & 0.52 & 0.35 & 1.43 & 0.47 & 0.39 & 1.16 & 0.35 & 0.31 & 0.38 & 0.22 & 0.15 & 1.43 & 0.42 & 0.39 \\
\hline $\mathrm{S} 66 \times 8$ & 1.36 & 0.46 & 0.32 & 1.39 & 0.48 & 0.34 & 1.10 & 0.39 & 0.30 & 0.38 & 0.23 & 0.16 & 1.39 & 0.35 & 0.34 \\
\hline F-set & 2.02 & 0.62 & 0.69 & 1.29 & 0.46 & 0.29 & 1.18 & 0.41 & 0.27 & 0.41 & 0.21 & 0.15 & 1.29 & 0.34 & 0.29 \\
\hline P-set & 1.80 & 0.52 & 0.43 & .85 & 0.44 & 0.35 & .83 & 0.38 & 0.24 & 0.39 & 0.17 & 0.14 & 0.85 & 0.36 & 0.35 \\
\hline S-set & 0.67 & 0.46 & 0.39 & 0.86 & 0.36 & 0.29 & 0.94 & 0.33 & 0.25 & 0.45 & 0.18 & 0.15 & 0.86 & 0.28 & 0.29 \\
\hline Cl-se & 2.18 & 0.43 & 0.44 & 0.73 & 0.46 & 0.39 & 0.81 & 0.45 & 0.31 & 0.39 & 0.19 & 0.16 & 0.73 & 0.39 & 0.39 \\
\hline B-set & 4.50 & 2.83 & 1.06 & 1.67 & 0.65 & 0.50 & 1.11 & 0.55 & 0.40 & 0.45 & 0.28 & 0.19 & 1.67 & 0.49 & 0.50 \\
\hline Si-set & 85 & 1.40 & 0.73 & 1.24 & 0.63 & 0.49 & 91 & 0.51 & 0.35 & 0.42 & 0.30 & .17 & 1.24 & 0.47 & 0.49 \\
\hline KB49 & 1.84 & 0.57 & 0.55 & 1.59 & 0.48 & 0.33 & 1.32 & 0.40 & 0.26 & 0.42 & 0.23 & 0.16 & 1.59 & 0.38 & 0.33 \\
\hline Water & 56.39 & 2.81 & 4.38 & 28.35 & 7.87 & 2.85 & 22.94 & 3.42 & 1.01 & 10.56 & 3.59 & 1.28 & 28.35 & 0.39 & 2.85 \\
\hline $\mathrm{A} 24$ & 93 & 0.45 & 0.52 & 1.12 & 0.49 & 0.36 & 0.78 & 0.29 & 0.21 & 0.32 & 0.22 & 0.15 & 1.12 & 0.33 & 0.36 \\
\hline HSG & 73 & 0.82 & 0.56 & 77 & 0.82 & & 25 & 0.61 & 0.26 & 23 & 0.19 & & 1.77 & 0.29 & 0.46 \\
\hline Ionic & 6.73 & 1.74 & 1.65 & .49 & 1.85 & 1.19 & 3.07 & 1.37 & 0.82 & 1.17 & 0.78 & 0.44 & 3.49 & 1.02 & 1.19 \\
\hline ADIM6 & 0.51 & 0.05 & 0.35 & 0.70 & 1.38 & 0.37 & 0.34 & 0.76 & 0.07 & 0.02 & 0.45 & 0.06 & 0.70 & 0.52 & 0.37 \\
\hline $\mathrm{X} 40 \times$ & 14 & 1.01 & 0.80 & 1 & 0.62 & 0. & 1.40 & 0.59 & 0.38 & 0.53 & 0.34 & 0.20 & 1.31 & 0.50 & 0.51 \\
\hline $\mathrm{S}$ & 75 & 0.33 & 0 . & 6 & 0.38 & 0 & 2 & 0.33 & 0.25 & 49 & 0.20 & 0 & .96 & 0.25 & 0.28 \\
\hline BBI & 1.85 & 0.93 & 0.49 & 80 & 0.48 & 0.17 & 1.42 & 0.38 & 0.15 & 0.27 & 0.18 & 0.09 & 1.80 & 0.58 & 0.17 \\
\hline SSI & 1.40 & 0.66 & 0.47 & 1.41 & 0.78 & 0 . & 0.96 & 0.52 & 0.25 & 0.18 & 0.19 & 0.10 & 1.41 & 0.33 & 0.37 \\
\hline $\mathrm{ACHC}$ & 0.84 & 1.21 & 0.51 & .79 & 0.30 & 0. & 1.82 & 0.17 & 0.38 & 0.56 & 0.47 & 0.22 & 1.79 & 0.19 & 0.33 \\
\hline HBC6 & 6.94 & 0.76 & 0.65 & 4 & 0.66 & 0 . & 2.43 & 0.71 & 0.36 & 0.37 & 0.23 & 0.14 & 2.74 & 0.49 & 0.65 \\
\hline NBC10ext & 0.89 & 0.48 & 0.39 & 83 & 0.43 & 0.28 & 0.65 & 0.31 & 0.21 & 0.26 & 0.15 & 0.11 & 0.83 & 0.25 & 0.28 \\
\hline Bauza & 5.80 & 13.78 & 15.29 & 1.94 & 3.38 & 2.3 & 2.92 & 4.60 & 2.47 & 1.19 & 1.45 & 0.81 & 1.94 & 3.93 & 2.37 \\
\hline P26 & 5.12 & 1.71 & 1.4 & 0.82 & 0.84 & 0. & 0.69 & 1.21 & 0.53 & 0.30 & 0.45 & 0.38 & 0.82 & 0.45 & 0.43 \\
\hline $\mathrm{ACO}$ & 1.30 & 0.93 & 0.62 & 0.12 & 0.54 & 0. & 0.13 & 0.21 & 0.28 & 0.04 & 0.22 & 0.16 & 0.12 & 0.41 & 0.47 \\
\hline CYCONF & 3.19 & 0.81 & 1.38 & 0.61 & 0.26 & 0.28 & 0.54 & 0.56 & 0.27 & 0.21 & 0.14 & 0.38 & 0.61 & 0.24 & 0.28 \\
\hline SCONF & 9.52 & 1.17 & 1.51 & 3.12 & 0.27 & 0.4 & 2.42 & 0.45 & 0.38 & 0.60 & 0.19 & 0.14 & 3.12 & 0.36 & 0.48 \\
\hline BCON & 4.62 & 0.30 & 0.43 & 8 & 0.30 & 0.49 & 1.67 & 0.25 & 0.39 & 0.84 & 0.35 & 0.17 & 1.88 & 0.45 & 0.49 \\
\hline BDE03 & 5.42 & 10.83 & 14.74 & 2.16 & 1.22 & 1.46 & 1.16 & 0.87 & 1.20 & 1.00 & 0.66 & 0.52 & 2.16 & 1.25 & 1.46 \\
\hline BHPeri & 13.38 & 10.19 & 5.15 & 3.63 & 0.97 & 1.28 & 2.01 & 1.06 & 0.76 & 1.74 & 0.59 & 0.37 & 3.63 & 1.35 & 1.28 \\
\hline BSR36 & 2.37 & 16.20 & 1.95 & 0.72 & 2.18 & 0.62 & 0.64 & 3.00 & 0.60 & 0.46 & 0.32 & 0.13 & 0.72 & 0.64 & 0.62 \\
\hline DARC & .36 & 30.78 & 19.38 & 8.03 & 1.69 & 0. & 4.08 & 2.54 & 0.71 & 4.03 & 0.58 & 0.35 & 8.03 & 0.68 & 0.65 \\
\hline BH76 & 19.09 & 13.03 & 11.82 & 4.26 & 3.67 & 2.33 & 3.14 & 2.90 & 1.44 & 0.97 & 0.83 & 0.52 & 4.26 & 2.51 & 2.33 \\
\hline ISO34 & 8.00 & 7.93 & 4.75 & 2.26 & 1.27 & 1.45 & 0.91 & 0.92 & 0.90 & 0.60 & 0.78 & 0.45 & 2.26 & 1.41 & 1.45 \\
\hline PA26 & 4.47 & 31.30 & 33.89 & 3.66 & 4.70 & 2.86 & 2.23 & 1.37 & 1.56 & 1.35 & 0.94 & 0.82 & 3.66 & 2.89 & 2.86 \\
\hline W4-1 & .55 & 46.39 & 81.79 & & 2.85 & 1.77 & 8.11 & 2.56 & 1.43 & 5.44 & 2.02 & 0.58 & 5.53 & 1.38 & 1.77 \\
\hline BDExrel & 4.53 & 1.27 & 0.99 & 0.64 & 0.67 & 0.44 & 0.72 & 0.48 & 0.30 & 0.61 & 0.22 & 0.14 & 0.64 & 0.49 & 0.44 \\
\hline
\end{tabular}


TABLE III. Mean absolute errors (MAE) for five basis sets (Def2-SV(P), Def2-SVP, pc-1, 6-31+G*, and 6-31+G**) in the individual subsets of the training set using B3LYP against the near-complete-basis-set reference (B3LYP/aug-cc-pVQZ). Bare $=$ the uncorrected basis set, LS = the least-squares fit prediction of the BSIP-corrected result, Eval = the actual BSIP result when used in a self-consistent calculation.

\begin{tabular}{|c|c|c|c|c|c|c|c|c|c|c|c|c|c|c|c|}
\hline & \multicolumn{3}{|c|}{ Def2-SV $(\mathrm{P})$} & \multicolumn{3}{|c|}{ 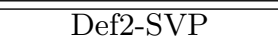 } & \multicolumn{3}{|c|}{$\overline{\mathrm{pc}-1}$} & \multicolumn{3}{|c|}{$6-31+\mathrm{G}^{*}$} & \multicolumn{3}{|c|}{$\overline{c 6-31+\mathrm{G}^{* *}}$} \\
\hline Set & Bare & LS & Eval & Bare & LS & Eval & Bare & LS & Eval & Bare & LS & Eval & Bare & $\mathrm{LS}$ & Eval \\
\hline $\mathrm{S} 22 \times 5$ & 1.60 & 0.44 & 0.42 & 1.58 & 0.38 & 0.35 & 1.16 & 0.32 & 0.31 & 0.42 & 0.17 & 0.18 & 0.38 & 0.13 & 0.15 \\
\hline $\mathrm{S} 66 \times 8$ & 1.63 & 0.44 & 0.44 & 1.57 & 0.37 & 0.37 & 1.10 & 0.32 & 0.30 & 0.42 & 0.19 & 0.20 & 0.38 & 0.15 & 0.16 \\
\hline F-set & 1.67 & 0.37 & 0.43 & 1.50 & 0.37 & 0.38 & 1.18 & 0.30 & 0.27 & 0.53 & 0.21 & 0.21 & 0.41 & 0.14 & 0.15 \\
\hline P-set & 1.21 & 0.51 & 0.57 & 1.17 & 0.47 & 0.59 & 0.83 & 0.25 & 0.24 & 0.42 & 0.20 & 0.19 & 0.39 & 0.13 & 0.14 \\
\hline S-set & 1.50 & 0.35 & 0.33 & 1.49 & 0.33 & 0.36 & 0.94 & 0.23 & 0.25 & 0.43 & 0.17 & 0.18 & 0.45 & 0.14 & 0.15 \\
\hline Cl-set & 1.51 & 0.49 & 0.48 & 1.45 & 0.46 & 0.46 & 0.81 & 0.32 & 0.31 & 0.38 & 0.21 & 0.21 & 0.39 & 0.16 & 0.16 \\
\hline B-set & 2.23 & 0.55 & 0.61 & 2.14 & 0.51 & 0.63 & 1.11 & 0.38 & 0.40 & 0.50 & 0.20 & 0.24 & 0.45 & 0.16 & 0.19 \\
\hline Si-set & 1.69 & 0.50 & 0.50 & 1.64 & 0.42 & 0.43 & 0.91 & 0.33 & 0.35 & 0.48 & 0.21 & 0.22 & 0.42 & 0.15 & 0.17 \\
\hline KB49 & 1.95 & 0.50 & 0.46 & 1.91 & 0.45 & 0.42 & 1.32 & 0.29 & 0.26 & 0.43 & 0.17 & 0.18 & 0.42 & 0.14 & 0.16 \\
\hline Water & 39.06 & 0.58 & 4.67 & 34.35 & 0.58 & 3.88 & 22.94 & 0.42 & 1.01 & 13.98 & 0.32 & 1.88 & 10.56 & 0.23 & 1.28 \\
\hline A24 & 1.27 & 0.32 & 0.40 & 1.25 & 0.33 & 0.43 & 0.78 & 0.22 & 0.21 & 0.37 & 0.15 & 0.16 & 0.32 & 0.14 & 0.15 \\
\hline HSG & 2.05 & 0.37 & 0.46 & 1.99 & 0.29 & 0.37 & 1.25 & 0.21 & 0.26 & 0.26 & 0.12 & 0.13 & 0.23 & 0.09 & 0.09 \\
\hline nicHB & 4.48 & 1.16 & 1.46 & 4.11 & 0.82 & 1.11 & 3.07 & 0.69 & 0.82 & 1.24 & 0.52 & 0.57 & 1.17 & 0.40 & 0.44 \\
\hline ADIM6 & 0.77 & 0.59 & 0.64 & 0.57 & 0.29 & 0.36 & 0.34 & 0.15 & 0.07 & 0.02 & 0.19 & 0.17 & .02 & 0.12 & 0.06 \\
\hline $\mathrm{X} 40 \times 1$ & 1.94 & 0.52 & 0.54 & 1.82 & 0.50 & 0.53 & 1.40 & 0.33 & 0.38 & 0.55 & 0.20 & 0.23 & 0.53 & 0.17 & 0.20 \\
\hline $\mathrm{S} \times 8$ & 1.65 & 0.35 & 0.38 & 1.64 & 0.33 & 0.38 & 1.02 & 0.23 & 0.25 & 0.50 & 0.19 & 0.21 & 0.49 & 0.16 & 0.18 \\
\hline BBI & 2.34 & 0.69 & 0.46 & 2.33 & 0.53 & 0.29 & 1.42 & 0.33 & 0.15 & 0.31 & 0.14 & 0 . & 0.27 & 0.09 & 0.09 \\
\hline SI & 50 & 0.31 & 0.39 & 1.49 & 0.27 & 0.38 & 0.96 & 0.22 & 0.25 & 0.18 & 0.13 & 0.12 & .18 & 0.10 & 0.10 \\
\hline $\mathrm{ACHC}$ & 2.40 & 0.20 & 0.30 & 2.45 & 0.21 & 0.41 & 1.82 & 0.15 & 0.38 & 0.57 & 0.09 & 0.26 & 0.56 & 0.08 & 0.22 \\
\hline HBC6 & 3.47 & 0.51 & 0.53 & 3.60 & 0.44 & 0.52 & 2.43 & 0.37 & 0.36 & 0.53 & 0.16 & 0.19 & 0.37 & 0.12 & 0.14 \\
\hline NBC10 & 0.75 & 0.33 & 0.36 & 0.75 & 0.28 & 0.33 & 0.65 & 0.20 & 0.21 & 0.20 & 0.11 & 0 . & 0.26 & 0.08 & 0.11 \\
\hline Bauza & 7.39 & 3.84 & 4.19 & 7.25 & 4.06 & 4.30 & 2.92 & 2.33 & 2.47 & 1.36 & 0.78 & 0.81 & 1.19 & 0.78 & 0.81 \\
\hline P26 & 0.99 & 0.61 & 0.59 & 0.97 & 0.53 & 0.49 & 0.69 & 0.48 & 0.53 & 0.34 & 0.44 & 0.45 & 0.30 & 0.37 & 0.38 \\
\hline $\mathrm{ACON}$ & 0.19 & 0.36 & 0.27 & 0.17 & 0.25 & 0.15 & 0.13 & 0.31 & 0.28 & 0.06 & 0.29 & 0.30 & 0.04 & 0.17 & 0.16 \\
\hline CYCONF & 1.01 & 0.35 & 0.32 & 1.15 & 0.19 & 0.15 & 0.54 & 0.28 & 0.27 & 0.18 & 0.52 & 0.50 & 0.21 & 0.39 & 0.38 \\
\hline SCONF & 3.12 & 0.41 & 0.56 & 2.88 & 0.28 & 0.35 & 2.42 & 0.36 & 0.38 & 1.01 & 0.21 & 0.17 & 0.60 & 0.15 & 0.14 \\
\hline BCONF & 2.74 & 0.42 & 0.47 & 2.42 & 0.37 & 0.50 & 1.67 & 0.38 & 0.39 & 1.12 & 0.15 & 0.22 & 0.84 & 0.11 & 0.17 \\
\hline BDE03 & 3.19 & 1.31 & 1.34 & 2.12 & 1.29 & 1.43 & 1.16 & 1.03 & 1.20 & 1.24 & 0.65 & 0.66 & 1.00 & 0.48 & 0.52 \\
\hline BHPeri & 3.14 & 1.39 & 1.51 & 2.74 & 0.99 & 1.09 & 2.01 & 0.78 & 0.76 & 2.04 & 0.54 & 0.55 & 1.74 & 0.36 & 0.37 \\
\hline BSR36 & 3.67 & 0.70 & 0.72 & 0.83 & 0.84 & 0.77 & 0.64 & 0.36 & 0.60 & 0.41 & 0.22 & 0.24 & 0.46 & 0.12 & 0.13 \\
\hline DARC & 8.20 & 0.75 & 0.98 & 7.17 & 0.91 & 0.88 & 4.08 & 0.45 & 0.71 & 4.91 & 0.37 & 0.48 & 4.03 & 0.22 & 0.35 \\
\hline BH76 & 4.28 & 2.21 & 2.47 & 3.61 & 2.04 & 2.25 & 3.14 & 1.45 & 1.44 & 1.45 & 0.59 & 0.60 & 0.97 & 0.49 & 0.52 \\
\hline ISO34 & 2.63 & 1.35 & 1.34 & 1.23 & 1.28 & 1.21 & 0.91 & 0.92 & 0.90 & 1.49 & 0.73 & 0.71 & 0.60 & 0.46 & 0.45 \\
\hline PA26 & 2.91 & 2.54 & 2.73 & 2.15 & 1.84 & 1.97 & 2.23 & 1.55 & 1.56 & 3.17 & 1.34 & 1.35 & 1.35 & 0.76 & 0.82 \\
\hline W4-11 & 6.49 & 1.37 & 1.78 & 3.97 & 1.31 & 1.79 & 8.11 & 0.99 & 1.43 & 7.37 & 0.62 & 0.76 & 5.44 & 0.48 & 0.58 \\
\hline BDExrel & 1.02 & 0.51 & 0.59 & 0.91 & 0.41 & 0.45 & 0.72 & 0.30 & 0.30 & 0.67 & 0.21 & 0.21 & 0.61 & 0.14 & 0.14 \\
\hline
\end{tabular}


TABLE IV. BSIP exponents $\left(\zeta_{l k}^{A}\right)$ and coefficients $\left(c_{l k}^{A}\right)$ for the MINI basis set.

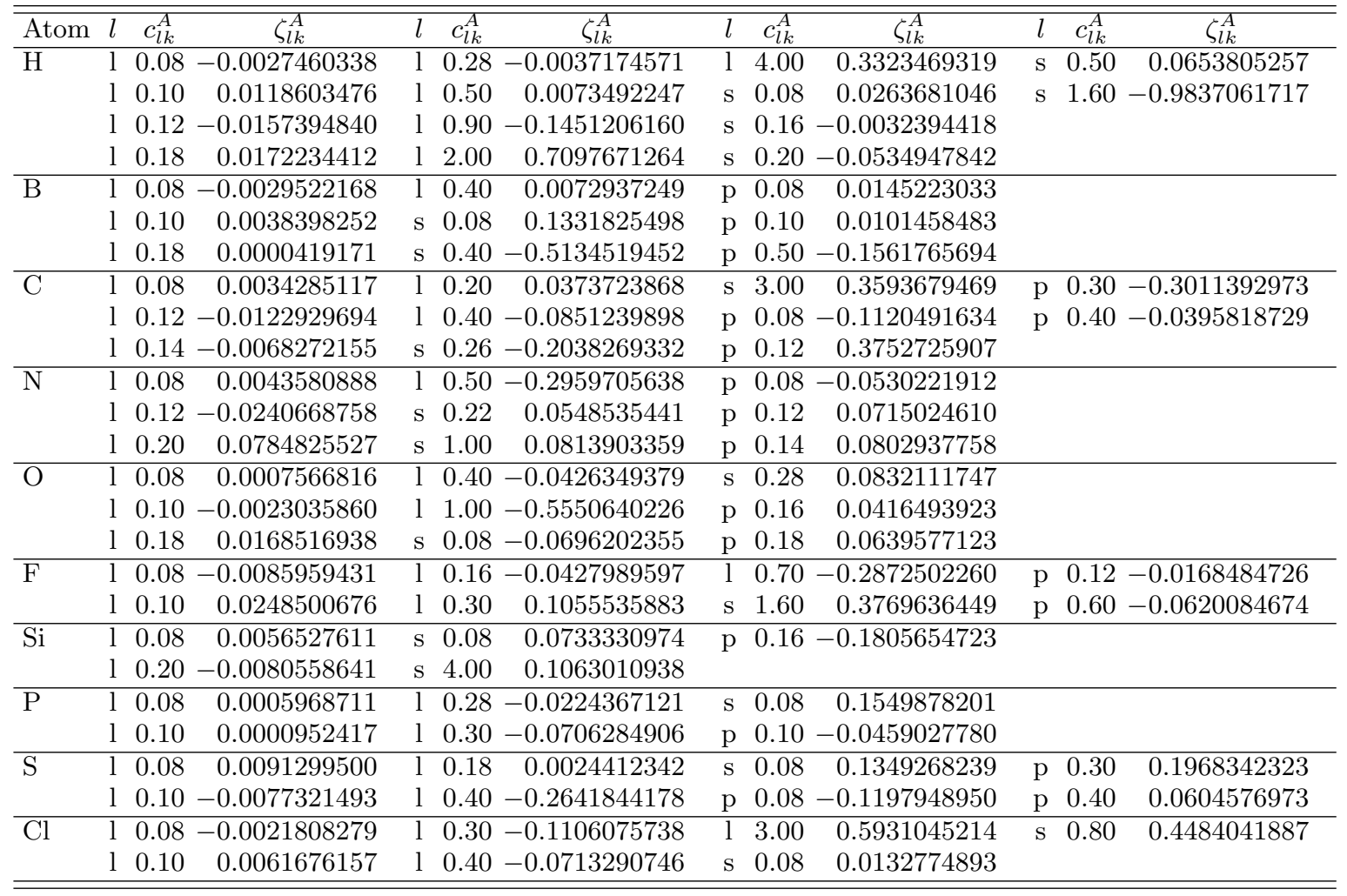


TABLE V. BSIP exponents $\left(\zeta_{l k}^{A}\right)$ and coefficients $\left(c_{l k}^{A}\right)$ for the MINIs basis set.

\begin{tabular}{|c|c|c|c|c|c|c|c|c|c|c|c|c|}
\hline Atom & $l$ & $\overline{c_{l k}^{A}}$ & $\zeta_{l k}^{A}$ & $l$ & $\bar{c} A$ & $\zeta_{l k}^{A}$ & $l$ & $\overline{c_{l k}^{A}}$ & $\zeta_{l k}^{A}$ & $l$ & $\overline{c_{l k}^{A}}$ & $\zeta_{l k}^{A}$ \\
\hline \multirow[t]{3}{*}{$\mathrm{H}$} & 1 & 0.08 & -0.0096022661 & 1 & 0.20 & 0.0837607141 & l & 4.00 & 1.1821111459 & $\mathrm{~s}$ & 0.50 & 0.0508091655 \\
\hline & 1 & 0.10 & 0.0551277062 & 1 & 0.30 & -0.0799978908 & $\mathrm{~s}$ & 0.08 & -0.0018353821 & $\mathrm{~s}$ & 0.60 & 0.5320275244 \\
\hline & 1 & 0.12 & -0.0753970714 & 1 & 0.70 & 0.0319967997 & $\mathrm{~s}$ & 0.18 & -0.0226244874 & $\mathrm{~s}$ & 1.80 & -1.8620021266 \\
\hline \multirow[t]{3}{*}{$\bar{B}$} & 1 & 0.08 & 0.0030796083 & 1 & 0.24 & -0.0316838819 & $\mathrm{~s}$ & 0.12 & 0.2396909850 & $\mathrm{p}$ & 0.08 & -0.0781571049 \\
\hline & 1 & 0.10 & -0.0009479500 & 1 & 0.50 & 0.0379450630 & $\mathrm{~s}$ & 0.50 & -0.3856815337 & $\mathrm{p}$ & 0.14 & 0.1517099090 \\
\hline & 1 & 0.22 & -0.0066285914 & 1 & 1.20 & -0.2522345343 & $\mathrm{~s}$ & 4.00 & 0.3212214974 & & & \\
\hline \multirow[t]{4}{*}{$\mathrm{C}$} & 1 & 0.08 & -0.0026180961 & 1 & 0.30 & -0.0589903412 & $\mathrm{~s}$ & 0.10 & 0.0264179594 & $\mathrm{p}$ & 0.14 & 0.1543425571 \\
\hline & 1 & 0.10 & 0.0291216037 & 1 & 0.40 & -0.1717704784 & $\mathrm{~s}$ & 0.30 & -0.0801191806 & $\mathrm{p}$ & 0.16 & 0.0054260032 \\
\hline & 1 & 0.12 & -0.0519307736 & 1 & 0.70 & 0.2890134877 & $\mathrm{~s}$ & 4.00 & 0.5135931759 & & & \\
\hline & 1 & 0.20 & 0.0819942824 & 1 & 1.60 & -0.6690791094 & $\mathrm{p}$ & 0.08 & -0.0410233758 & & & \\
\hline \multirow[t]{3}{*}{$\mathrm{N}$} & $\mathrm{l}$ & 0.08 & 0.0039160890 & l & 0.22 & 0.0086803679 & $\mathrm{~s}$ & 0.10 & 0.0731819053 & $\mathrm{p}$ & 0.14 & 0.2438428886 \\
\hline & l & 0.12 & -0.0120960452 & 1 & 0.70 & -0.3205701579 & $\mathrm{~s}$ & 0.50 & -0.0414268056 & $\mathrm{p}$ & 0.50 & -0.0906861563 \\
\hline & l & 0.20 & 0.0070526283 & 1 & 3.00 & 0.2883822236 & $\mathrm{p}$ & 0.08 & -0.0874270479 & & & \\
\hline \multirow[t]{4}{*}{$\mathrm{O}$} & l & 0.08 & -0.0252132532 & 1 & 0.40 & -0.0621843563 & $\mathrm{~s}$ & 4.00 & 0.0076575871 & $\mathrm{p}$ & 0.50 & -0.0985396585 \\
\hline & 1 & 0.10 & 0.1154773506 & 1 & 1.00 & -0.4934296935 & $\mathrm{p}$ & 0.08 & -0.0441084329 & $p$ & 4.00 & 0.5474151274 \\
\hline & l & 0.12 & -0.1313492750 & $\mathrm{~s}$ & 0.08 & 0.0518394716 & $\mathrm{p}$ & 0.14 & 0.0104554608 & & & \\
\hline & 1 & 0.20 & 0.0703951469 & $\mathrm{~s}$ & 0.40 & -0.0770530214 & $\mathrm{p}$ & 0.16 & 0.1378003772 & & & \\
\hline \multirow[t]{3}{*}{$\bar{F}$} & $\bar{l}$ & 0.08 & -0.0076510181 & $\mathrm{l}$ & 0.28 & 0.0544536559 & $\mathrm{l}$ & 0.80 & -0.2184186297 & $\mathrm{p}$ & 0.18 & 0.0553750681 \\
\hline & l & 0.10 & 0.0222238200 & 1 & 0.30 & 0.0558704797 & $\mathrm{~s}$ & 0.08 & -0.0304926853 & & & \\
\hline & l & 0.16 & -0.0450380877 & 1 & 0.70 & -0.2692779501 & $\mathrm{~s}$ & 4.00 & 0.0825775194 & & & \\
\hline \multirow[t]{2}{*}{$\mathrm{Si}$} & 1 & 0.08 & 0.0054118548 & 1 & 0.20 & -0.0390870511 & $\mathrm{p}$ & 0.18 & 0.0603591119 & & & \\
\hline & l & 0.18 & -0.0000522598 & $\mathrm{~s}$ & 0.08 & -0.1539039298 & $\mathrm{p}$ & 3.00 & 0.4430728735 & & & \\
\hline \multirow[t]{2}{*}{$\mathrm{P}$} & 1 & 0.08 & 0.0020792129 & 1 & 0.26 & -0.0619569394 & $\mathrm{~s}$ & 0.08 & 0.1021454874 & $\mathrm{p}$ & 0.20 & 0.1155644567 \\
\hline & l & 0.14 & -0.0022228686 & 1 & 0.28 & -0.0316967823 & $\mathrm{p}$ & 0.08 & -0.0542945446 & & & \\
\hline \multirow[t]{3}{*}{ S } & 1 & 0.08 & 0.0040854522 & 1 & 0.40 & -0.2787783137 & $\mathrm{~s}$ & 4.00 & -0.2989405707 & $\mathrm{p}$ & 0.26 & 0.6618928120 \\
\hline & l & 0.12 & -0.0019571135 & 1 & 0.50 & -0.0496888076 & $\mathrm{p}$ & 0.08 & -0.0477563007 & & & \\
\hline & l & 0.22 & 0.0040959892 & $\mathrm{~s}$ & 0.08 & 0.2903200571 & $\mathrm{p}$ & 0.10 & -0.2822775036 & & & \\
\hline \multirow[t]{2}{*}{$\mathrm{Cl}$} & 1 & 0.08 & 0.0034742053 & 1 & 0.16 & 0.0317393925 & $\mathrm{~s}$ & 0.12 & 0.0420597602 & $\mathrm{p}$ & $\overline{0.12}$ & 0.1074338340 \\
\hline & l & 0.10 & -0.0099282374 & 1 & 0.30 & -0.2030771775 & $\mathrm{p}$ & 0.08 & -0.0473742947 & & & \\
\hline
\end{tabular}

TABLE VI. BSIP exponents $\left(\zeta_{l k}^{A}\right)$ and coefficients $\left(c_{l k}^{A}\right)$ for the STO-3G basis set.

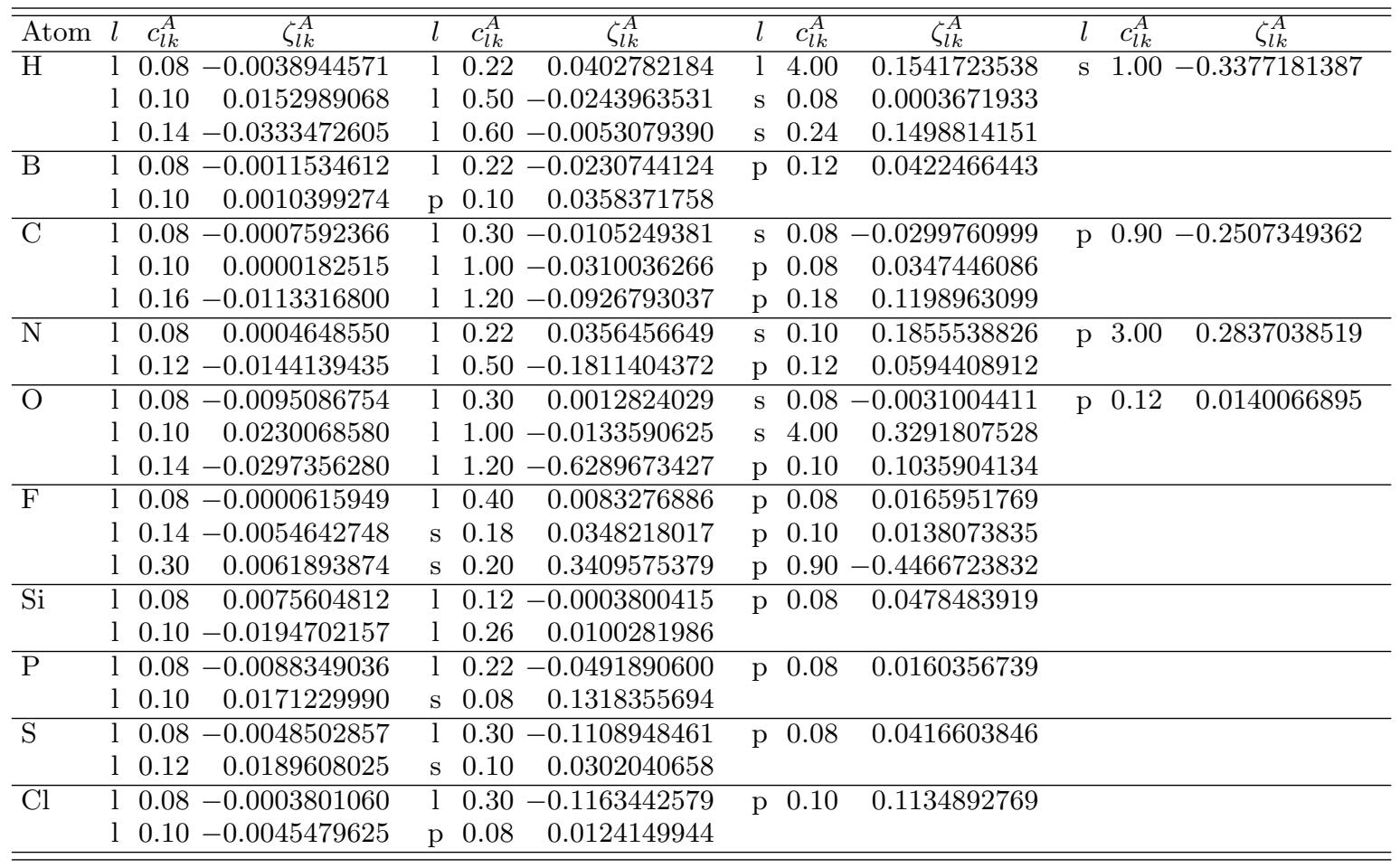


TABLE VII. BSIP exponents $\left(\zeta_{l k}^{A}\right)$ and coefficients $\left(c_{l k}^{A}\right)$ for the $3-21 \mathrm{G}$ basis set.

\begin{tabular}{|c|c|c|c|c|c|c|c|c|c|c|}
\hline Atom & $l$ & 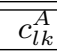 & $\overline{\zeta_{l k}^{A}}$ & $l$ & $\overline{c_{l k}^{A}}$ & $\overline{\zeta_{l k}^{A}}$ & $l$ & $\bar{c} c_{l k}^{A}$ & $\overline{\zeta_{l k}^{A}}$ & $\begin{array}{ll}l \quad c_{l k}^{A} \\
\end{array}$ \\
\hline \multirow[t]{3}{*}{$\mathrm{H}$} & l & 0.08 & -0.0018884000 & l & 0.18 & 0.0204928025 & l & 0.60 & 0.0945161521 & s $1.80-0.4356272116$ \\
\hline & 1 & 0.10 & 0.0160354960 & 1 & 0.20 & 0.0089184125 & $\mathrm{~s}$ & 0.08 & -0.0188007453 & \\
\hline & 1 & 0.12 & -0.0259498830 & 1 & 0.30 & -0.0535148391 & $\mathrm{~s}$ & 0.16 & 0.0851543773 & \\
\hline \multirow[t]{2}{*}{$\bar{B}$} & $\mathrm{l}$ & 0.08 & 0.0018072913 & 1 & 0.60 & -0.0158031067 & $\mathrm{~s}$ & 3.00 & -0.0141455591 & 0.0034873339 \\
\hline & 1 & 0.14 & -0.0152548951 & $\mathrm{~s}$ & 0.08 & 0.0094512135 & $\mathrm{p}$ & 0.14 & 0.0590706224 & \\
\hline \multirow[t]{3}{*}{$\mathrm{C}$} & 1 & 0.08 & 0.0002972085 & l & 0.30 & -0.0243500000 & $\mathrm{p}$ & 0.08 & 0.0199401615 & \\
\hline & 1 & 0.10 & -0.0032009971 & 1 & 2.00 & -0.0363956991 & $\mathrm{p}$ & 0.26 & 0.0352533309 & \\
\hline & 1 & 0.14 & 0.0022457582 & $\mathrm{~s}$ & 0.10 & -0.0098607350 & $\mathrm{p}$ & 0.28 & 0.0228951552 & \\
\hline \multirow[t]{3}{*}{$\mathrm{N}$} & 1 & $0.0 \varepsilon$ & -0.0003142792 & l & 0.40 & -0.0436318077 & $\mathrm{p}$ & 0.08 & -0.0172014382 & \\
\hline & 1 & 0.10 & -0.0020347169 & $\mathrm{~s}$ & 0.08 & 0.1232943572 & $\mathrm{p}$ & 0.14 & 0.0539848500 & \\
\hline & 1 & 0.18 & 0.0001744891 & $\mathrm{~s}$ & 0.40 & -0.1642169777 & $\mathrm{p}$ & 3.00 & 0.0577254721 & \\
\hline \multirow[t]{3}{*}{$\mathrm{O}$} & 1 & 0.08 & -0.0034393050 & l & 0.50 & 0.0138763057 & $\mathrm{~s}$ & 0.50 & -0.3602906151 & p $0.50-0.3664441015$ \\
\hline & 1 & 0.10 & 0.0039875365 & $\mathrm{~s}$ & 0.08 & 0.0990296566 & $\mathrm{p}$ & 0.08 & 0.0047730392 & 0.9299612971 \\
\hline & 1 & 0.20 & -0.0033979966 & $\mathrm{~s}$ & 0.40 & -0.2556928697 & $\mathrm{p}$ & 0.10 & 0.0255397377 & \\
\hline \multirow[t]{3}{*}{$\overline{\mathrm{F}}$} & l & 0.08 & -0.0079356075 & l & 0.30 & 0.0234125499 & $\mathrm{~s}$ & 4.00 & 0.1732705334 & 0.0499877123 \\
\hline & l & 0.10 & 0.0196179646 & l & 0.80 & -0.1067993960 & $\mathrm{p}$ & 0.08 & 0.0228667206 & \\
\hline & l & 0.16 & -0.0169858553 & 1 & 0.90 & -0.0676893781 & $\mathrm{p}$ & 0.50 & -0.0918492323 & \\
\hline \multirow[t]{2}{*}{$\mathrm{Si}$} & l & 0.08 & -0.0053779989 & l & 0.22 & -0.0376524671 & $\mathrm{~s}$ & 0.08 & 0.0136173110 & \\
\hline & l & 0.10 & 0.0041071956 & 1 & 0.24 & -0.0236539794 & $\mathrm{p}$ & 0.08 & 0.1298616968 & \\
\hline \multirow[t]{2}{*}{$\overline{\mathrm{P}}$} & 1 & 0.08 & 0.0058606811 & l & 0.30 & -0.0282782849 & $\mathrm{~s}$ & 3.00 & 0.1234792660 & \\
\hline & l & 0.10 & -0.0100607938 & 1 & 0.40 & -0.0129427836 & & & & \\
\hline \multirow[t]{2}{*}{$\bar{S}$} & 1 & 0.08 & 0.0029980678 & l & 0.14 & -0.0016003038 & $\mathrm{~s}$ & 0.60 & 0.0224119492 & \\
\hline & l & 0.12 & -0.0112169782 & 1 & 0.40 & -0.1362594268 & $\mathrm{p}$ & 0.26 & 0.1277521546 & \\
\hline \multirow[t]{2}{*}{$\mathrm{Cl}$} & 1 & 0.08 & -0.0021910859 & l & 0.22 & -0.0001209882 & $\mathrm{p}$ & 0.12 & 0.0063443466 & \\
\hline & l & 0.10 & 0.0065850505 & 1 & 0.24 & -0.0694481265 & $\mathrm{p}$ & 0.14 & 0.0750244363 & \\
\hline
\end{tabular}

TABLE VIII. BSIP exponents $\left(\zeta_{l k}^{A}\right)$ and coefficients $\left(c_{l k}^{A}\right)$ for the $6-31 \mathrm{G}$ basis set.

\begin{tabular}{|c|c|c|c|c|c|c|c|c|c|c|c|c|}
\hline Atom & $\overline{l l}$ & $c_{l k}^{A}$ & $\zeta_{l k}^{A}$ & $l$ & $\bar{c} c_{l k}^{A}$ & $\zeta_{l k}^{A}$ & $\bar{l}$ & $c_{l k}^{A}$ & $\overline{\zeta_{l k}^{A}}$ & $\bar{l}$ & $c_{l k}^{A}$ & $\overline{\zeta_{l k}^{A}}$ \\
\hline \multirow[t]{3}{*}{$\mathrm{H}$} & l & 0.08 & -0.0016306959 & 1 & 0.18 & 0.0247596086 & $\mathrm{~s}$ & 0.08 & -0.0027475600 & $\mathrm{~s}$ & 2.00 & -0.3446458212 \\
\hline & 1 & 0.10 & 0.0130601738 & 1 & 0.30 & -0.0375467464 & $\mathrm{~s}$ & 0.12 & 0.0055865931 & & & \\
\hline & 1 & 0.12 & -0.0222082847 & 1 & 0.60 & 0.0563703198 & $\mathrm{~s}$ & 0.40 & 0.0702849529 & & & \\
\hline \multirow[t]{2}{*}{ B } & l & 0.08 & 0.0006465852 & 1 & 0.14 & -0.0030833419 & $\mathrm{~s}$ & 0.10 & 0.0718748718 & $\mathrm{p}$ & 0.20 & 0.0390267363 \\
\hline & 1 & 0.12 & -0.0042987523 & 1 & 0.60 & -0.0489379424 & $\mathrm{p}$ & 0.18 & 0.0113676738 & & & \\
\hline \multirow[t]{3}{*}{$\mathrm{C}$} & l & 0.08 & -0.0002723924 & 1 & 0.22 & 0.0084363350 & $\mathrm{~s}$ & 0.08 & -0.0163846037 & $\mathrm{p}$ & 0.60 & 0.0851420352 \\
\hline & 1 & 0.12 & -0.0025351769 & 1 & 0.50 & -0.0015054181 & $\mathrm{~s}$ & 0.30 & 0.0301326659 & & & \\
\hline & 1 & 0.20 & -0.0055970529 & 1 & 0.60 & -0.0699592848 & $\mathrm{p}$ & 0.08 & 0.0212299126 & & & \\
\hline \multirow[t]{3}{*}{$\mathrm{N}$} & l & 0.08 & 0.0016102930 & l & 0.24 & -0.0110792668 & $\mathrm{~s}$ & 0.30 & -0.1565847716 & $\mathrm{p}$ & 1.40 & 0.0145990614 \\
\hline & 1 & 0.12 & -0.0053984387 & l & 0.70 & -0.1002787483 & $\mathrm{p}$ & 0.08 & -0.0826338988 & & & \\
\hline & 1 & 0.22 & -0.0003073135 & $\mathrm{~s}$ & 0.08 & 0.1722946988 & $\mathrm{p}$ & 0.16 & 0.1753189309 & & & \\
\hline \multirow[t]{3}{*}{$\mathrm{O}$} & l & 0.08 & -0.0004726872 & 1 & 0.30 & -0.0373583680 & $\mathrm{p}$ & 0.08 & -0.0318778747 & $\mathrm{p}$ & 4.00 & 0.6121920499 \\
\hline & 1 & 0.12 & -0.0039255168 & $\mathrm{~s}$ & 0.08 & 0.1725024077 & $\mathrm{p}$ & 0.16 & -0.0337882306 & & & \\
\hline & 1 & 0.18 & 0.0185607000 & $\mathrm{~s}$ & 0.60 & -0.6496913416 & $\mathrm{p}$ & 0.30 & -0.0337493838 & & & \\
\hline \multirow[t]{3}{*}{$\bar{F}$} & l & 0.08 & -0.0045895849 & 1 & 0.16 & -0.0016493501 & $\mathrm{p}$ & 0.08 & 0.0266551696 & & & \\
\hline & 1 & 0.10 & 0.0108367691 & 1 & 0.70 & -0.0555029738 & $\mathrm{p}$ & 0.50 & -0.0643289886 & & & \\
\hline & 1 & 0.14 & -0.0064611485 & $\mathrm{~s}$ & 0.08 & 0.0006332640 & $\mathrm{p}$ & 4.00 & 0.0106677455 & & & \\
\hline \multirow[t]{2}{*}{$\mathrm{Si}$} & l & 0.08 & -0.0020580253 & 1 & 0.18 & 0.0005884184 & $\mathrm{p}$ & 0.08 & 0.0875008137 & & & \\
\hline & 1 & 0.10 & -0.0042904241 & 1 & 0.30 & -0.0690073429 & & & & & & \\
\hline \multirow[t]{2}{*}{$\mathrm{P}$} & 1 & 0.08 & 0.0021620814 & 1 & 0.18 & 0.0019703165 & $\mathrm{~s}$ & 0.08 & 0.0182621649 & $\mathrm{p}$ & 0.50 & 0.0391591774 \\
\hline & 1 & 0.10 & -0.0052293736 & 1 & 0.40 & -0.1228386283 & $\mathrm{p}$ & 0.40 & 0.0494966314 & & & \\
\hline \multirow[t]{2}{*}{$\mathrm{S}$} & l & 0.08 & 0.0028771287 & 1 & 0.24 & 0.0081482688 & $\mathrm{~s}$ & 0.40 & 0.2924424974 & $\mathrm{p}$ & 0.40 & 0.0267448068 \\
\hline & 1 & 0.12 & -0.0150345308 & 1 & 0.50 & -0.3349033900 & $\mathrm{p}$ & 0.30 & 0.2320405651 & & & \\
\hline \multirow[t]{2}{*}{$\mathrm{Cl}$} & l & 0.08 & -0.0000801597 & 1 & 0.22 & -0.0046989063 & l & 0.40 & -0.1237646265 & $\mathrm{p}$ & 0.10 & 0.0211832705 \\
\hline & 1 & 0.10 & -0.0008577930 & 1 & 0.24 & -0.0160793867 & $\mathrm{~s}$ & 0.14 & 0.0129224955 & $\mathrm{p}$ & 0.18 & 0.0896259150 \\
\hline
\end{tabular}


TABLE IX. BSIP exponents $\left(\zeta_{l k}^{A}\right)$ and coefficients $\left(c_{l k}^{A}\right)$ for the MIDIh basis set.

\begin{tabular}{|c|c|c|c|c|c|c|c|c|c|c|c|c|}
\hline Atom & $l$ & $\overline{c_{l k}^{A}}$ & $\zeta_{l k}^{A}$ & $l$ & $c_{l k}^{A}$ & $\zeta_{l k}^{A}$ & $l$ & $\overline{c_{l k}^{A}}$ & $\zeta_{l k}^{A}$ & $l$ & $c_{l k}^{A}$ & $\zeta_{l k}^{A}$ \\
\hline \multirow[t]{3}{*}{$\mathrm{H}$} & 1 & 0.08 & 0.0015493709 & 1 & 0.18 & 0.0291599835 & $\mathrm{~S}$ & 0.08 & -0.0172159730 & $\mathrm{~s}$ & 2.00 & -0.3165102637 \\
\hline & 1 & 0.10 & 0.0027161840 & 1 & 0.30 & -0.0670361395 & $S$ & 0.20 & 0.1005100335 & & & \\
\hline & l & 0.12 & -0.0135292893 & 1 & 0.60 & 0.1139896906 & $\mathrm{~s}$ & 1.80 & -0.2246863821 & & & \\
\hline \multirow[t]{2}{*}{$\mathrm{B}$} & 1 & 0.08 & 0.0034536691 & 1 & 0.40 & -0.0233751920 & $\mathrm{p}$ & 0.18 & 0.0451520405 & & & \\
\hline & l & 0.12 & -0.0120581474 & $\mathrm{~S}$ & 4.00 & -0.0001513283 & $\mathrm{p}$ & 0.20 & 0.0303037804 & & & \\
\hline \multirow[t]{2}{*}{$\mathrm{C}$} & 1 & 0.08 & -0.0007707052 & l & 0.12 & -0.0020517925 & l & 0.40 & 0.0117748449 & $\mathrm{p}$ & 0.08 & 0.0198474471 \\
\hline & l & 0.10 & 0.0015587948 & 1 & 0.30 & -0.0299537184 & $\mathrm{~s}$ & 0.10 & -0.0290357574 & $\mathrm{p}$ & 0.40 & 0.0609850897 \\
\hline \multirow[t]{3}{*}{$\mathrm{N}$} & l & 0.08 & 0.0065096436 & 1 & 0.30 & -0.0450252246 & $\mathrm{p}$ & 0.08 & -0.1163451535 & & & \\
\hline & 1 & 0.10 & -0.0168223452 & $\mathrm{~S}$ & 0.08 & 0.1441047718 & $p$ & 0.10 & 0.1396796771 & & & \\
\hline & 1 & 0.16 & 0.0230454795 & $\mathrm{~S}$ & 0.30 & -0.1687102290 & $\mathrm{p}$ & 1.60 & 0.0069043196 & & & \\
\hline \multirow[t]{2}{*}{$\mathrm{O}$} & l & 0.08 & -0.0044461262 & 1 & 0.20 & -0.0047085373 & $\mathrm{~S}$ & 0.50 & -0.5504334166 & $\mathrm{p}$ & 0.50 & -0.2753391579 \\
\hline & l & 0.10 & 0.0058983846 & $\mathrm{~S}$ & 0.08 & 0.1215185059 & $\mathrm{p}$ & 0.10 & 0.0146835101 & $\mathrm{p}$ & 4.00 & 0.6431185590 \\
\hline \multirow[t]{2}{*}{$\mathrm{F}$} & 1 & 0.08 & -0.0048258353 & 1 & 0.18 & -0.0024020361 & $\mathrm{~S}$ & 4.00 & 0.1213184773 & $\mathrm{p}$ & 0.50 & -0.1275278294 \\
\hline & l & 0.10 & 0.0113590911 & 1 & 0.70 & -0.0648676636 & $\mathrm{p}$ & 0.08 & 0.0178104858 & & & \\
\hline \multirow[t]{2}{*}{$\mathrm{Si}$} & l & 0.08 & 0.0070418371 & 1 & 0.20 & -0.0528271869 & $\mathrm{p}$ & 0.40 & 0.1546341761 & & & \\
\hline & l & 0.18 & -0.0240867035 & $\mathrm{p}$ & 0.08 & 0.1159332835 & & & & & & \\
\hline \multirow[t]{2}{*}{$\mathrm{P}$} & 1 & 0.08 & 0.0079715380 & 1 & 0.16 & 0.0136136021 & l & 0.40 & -0.0815163912 & $\mathrm{~s}$ & 0.60 & 0.0054847045 \\
\hline & 1 & 0.10 & -0.0175623207 & 1 & 0.30 & -0.0138920224 & $\mathrm{~s}$ & 0.08 & 0.0217045963 & $\mathrm{p}$ & 0.08 & 0.0042473886 \\
\hline \multirow[t]{2}{*}{$\mathrm{S}$} & 1 & 0.08 & -0.0028793854 & 1 & 0.30 & -0.0879294334 & $\mathrm{p}$ & 0.08 & 0.0662729417 & $\mathrm{p}$ & 0.80 & 0.2121059150 \\
\hline & 1 & 0.12 & 0.0020305247 & 1 & 0.40 & -0.1095917497 & $\mathrm{p}$ & 0.70 & 0.1347823638 & & & \\
\hline \multirow[t]{2}{*}{$\mathrm{Cl}$} & l & 0.08 & -0.0000487050 & 1 & 0.26 & -0.0002085163 & $\mathrm{p}$ & 0.14 & 0.1066727189 & $\mathrm{p}$ & 0.50 & 0.0195232426 \\
\hline & l & 0.10 & 0.0009427402 & 1 & 0.28 & -0.1230966256 & $\mathrm{p}$ & 0.40 & 0.0780743055 & & & \\
\hline
\end{tabular}

TABLE X. BSIP exponents $\left(\zeta_{l k}^{A}\right)$ and coefficients $\left(c_{l k}^{A}\right)$ for the pc-0 basis set.

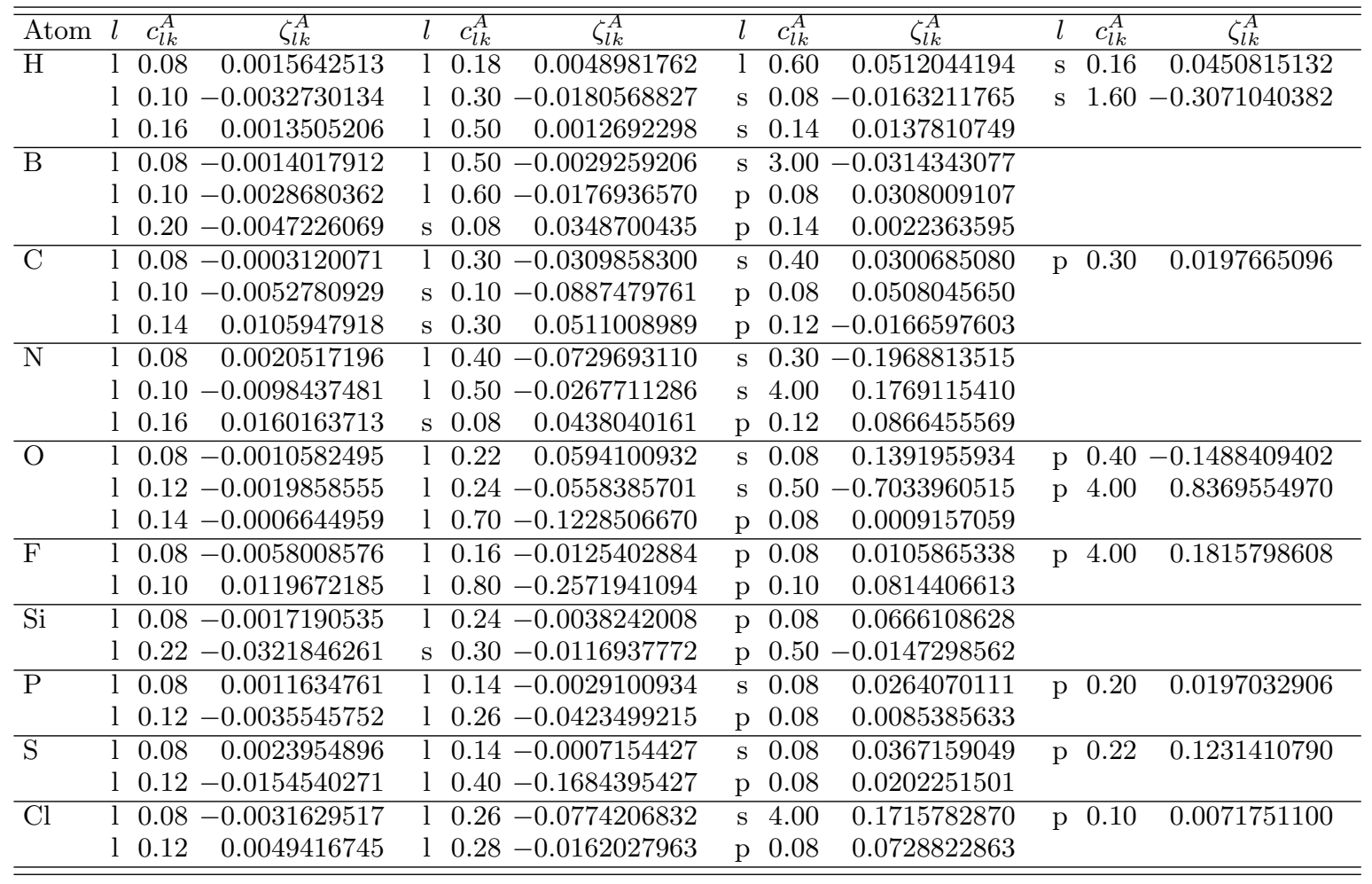


TABLE XI. BSIP exponents $\left(\zeta_{l k}^{A}\right)$ and coefficients $\left(c_{l k}^{A}\right)$ for the $6-31+\mathrm{G}$ basis set.

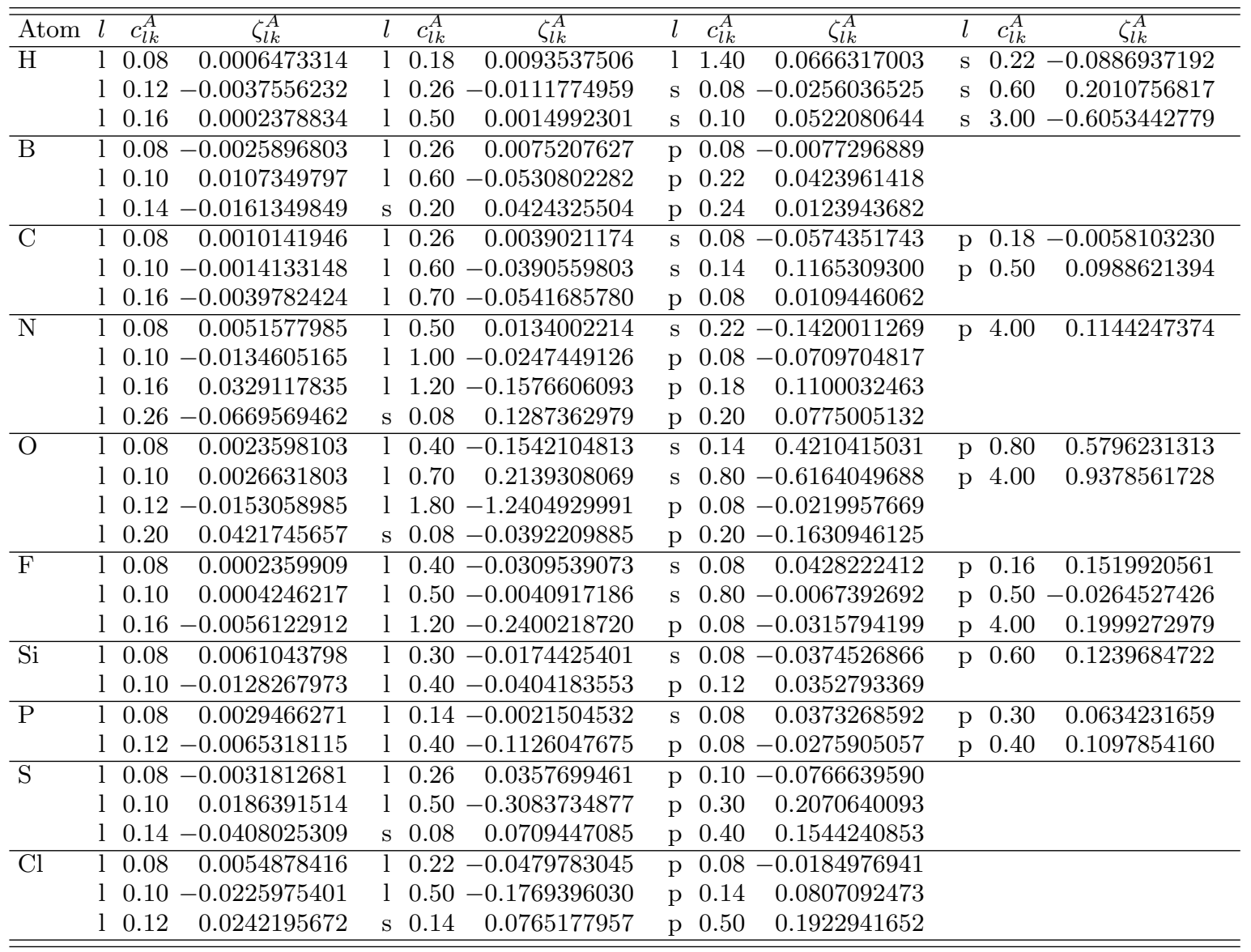

TABLE XII. BSIP exponents $\left(\zeta_{l k}^{A}\right)$ and coefficients $\left(c_{l k}^{A}\right)$ for the $3-21 \mathrm{G}^{*}$ basis set.

\begin{tabular}{|c|c|c|c|c|c|c|c|c|c|c|c|c|}
\hline Atom & $l$ & $\overline{\overline{c_{l k}^{A}}}$ & $\overline{\zeta_{l k}^{A}}$ & $l$ & $\overline{c_{l k}^{A}}$ & $\overline{\zeta_{l k}^{A}}$ & $l$ & $\overline{c_{l k}^{A}}$ & $\overline{\zeta_{l k}^{A}}$ & $l$ & $\overline{c_{l k}^{A}}$ & $\overline{\zeta_{l k}^{A}}$ \\
\hline \multirow[t]{3}{*}{$\mathrm{H}$} & $\bar{l}$ & 0.08 & -0.0008241823 & 1 & 0.20 & 0.0252006574 & 1 & 0.70 & 0.0990287121 & $\mathrm{~s}$ & 0.22 & 0.0131846023 \\
\hline & 1 & 0.10 & 0.0093213416 & 1 & 0.30 & -0.0451953860 & $\mathrm{~s}$ & 0.08 & -0.0058409035 & $\mathrm{~s}$ & 2.00 & -0.3364789964 \\
\hline & 1 & 0.12 & -0.0157978449 & 1 & 0.60 & 0.0032651573 & $\mathrm{~s}$ & 0.20 & 0.0550690696 & $\mathrm{~s}$ & 3.00 & -0.2339459672 \\
\hline \multirow[t]{2}{*}{ B } & 1 & 0.08 & 0.0016413670 & 1 & 0.16 & -0.0071194932 & $\mathrm{p}$ & 0.12 & 0.0134801005 & $\mathrm{~d}$ & 0.10 & 0.0032459986 \\
\hline & l & 0.14 & -0.0105144705 & l & 0.40 & -0.0060626103 & $\mathrm{p}$ & 0.14 & 0.0528919981 & & & \\
\hline \multirow[t]{3}{*}{$\mathrm{C}$} & l & 0.08 & 0.0004166075 & l & 0.16 & 0.0056198653 & $\mathrm{~s}$ & 0.10 & -0.0043803525 & $\mathrm{p}$ & 0.28 & 0.0101649717 \\
\hline & 1 & 0.10 & -0.0038168528 & 1 & 0.30 & -0.0450396817 & $p$ & 0.08 & 0.0193917542 & $\mathrm{~d}$ & 0.08 & 0.0064574910 \\
\hline & 1 & 0.1 & 0.0005628850 & $\mathrm{~S}$ & 0.08 & -0.0250104796 & $\mathrm{p}$ & 0.26 & 0.0584550088 & d & 0.40 & 0.0833039807 \\
\hline \multirow[t]{3}{*}{$\mathrm{N}$} & l & 0.08 & 0.0003916001 & 1 & 0.40 & -0.0164077218 & $\mathrm{~s}$ & 0.30 & -0.1664132099 & $\mathrm{~d}$ & 0.08 & -0.0388663551 \\
\hline & 1 & 0.12 & -0.0003538219 & 1 & 0.50 & -0.0284506715 & $\mathrm{p}$ & 0.10 & 0.0321255805 & $\mathrm{~d}$ & 0.12 & 0.0073801594 \\
\hline & 1 & 0.14 & -0.0063469357 & $\mathrm{~s}$ & 0.08 & 0.1205777630 & $\mathrm{p}$ & 4.00 & 0.0502915373 & d & 0.14 & 0.0613485431 \\
\hline \multirow[t]{3}{*}{$\mathrm{O}$} & l & 0.08 & -0.0039608146 & l & 0.40 & 0.0361862165 & $\mathrm{~s}$ & 0.50 & -0.0915865082 & $\mathrm{p}$ & 4.00 & 0.8168585647 \\
\hline & 1 & 0.10 & 0.0053825662 & $\mathrm{~s}$ & 0.08 & 0.1148475966 & $\mathrm{p}$ & 0.10 & 0.0621323947 & d & 0.10 & -0.0272332647 \\
\hline & 1 & 0.16 & -0.0031536703 & $\mathrm{~S}$ & 0.40 & -0.5546976510 & $\mathrm{p}$ & 0.40 & -0.3965171927 & $\mathrm{~d}$ & 0.50 & 0.0461667070 \\
\hline \multirow[t]{3}{*}{$\mathrm{F}$} & 1 & 0.08 & -0.0078702670 & 1 & 0.30 & 0.0245983657 & $\mathrm{p}$ & 0.08 & 0.0130720789 & d & 0.50 & 0.3000635958 \\
\hline & 1 & 0.10 & 0.0189012629 & 1 & 0.80 & -0.2568645121 & d & 0.08 & 0.0067142240 & & & \\
\hline & 1 & 0.18 & -0.0239171771 & 1 & 0.90 & -0.0306783119 & d & 0.40 & 0.0100023205 & & & \\
\hline \multirow[t]{2}{*}{$\mathrm{Si}$} & 1 & 0.08 & -0.0034823089 & 1 & 0.20 & -0.0141337933 & $p$ & 0.10 & 0.0239583539 & d & 0.14 & 0.0479902246 \\
\hline & 1 & 0.18 & -0.0301914690 & $\mathrm{p}$ & 0.08 & 0.1114359871 & d & 0.12 & 0.0136248005 & & & \\
\hline $\mathrm{P}$ & 1 & 0.08 & 0.0072422588 & l & 0.10 & -0.0159492430 & 1 & 0.18 & 0.0170885982 & $\mathrm{p}$ & 0.08 & -0.0044265894 \\
\hline \multirow[t]{2}{*}{$\mathrm{S}$} & l & 0.08 & 0.0038575830 & l & 0.30 & 0.0123906057 & $\mathrm{p}$ & 0.08 & 0.0155536448 & & & \\
\hline & 1 & 0.10 & -0.0094131156 & $\mathrm{~S}$ & 0.08 & -0.0183255172 & d & 0.10 & -0.0064713097 & & & \\
\hline \multirow[t]{2}{*}{$\mathrm{Cl}$} & l & 0.08 & -0.0014861761 & l & 0.18 & -0.0073777229 & $\mathrm{p}$ & 0.08 & 0.0021032738 & $\mathrm{~d}$ & 0.30 & 0.0031029226 \\
\hline & l & 0.10 & 0.0052324580 & $\mathrm{~s}$ & 0.40 & 0.0313439367 & $p$ & 0.22 & 0.0212285210 & & & \\
\hline
\end{tabular}


TABLE XIII. BSIP exponents $\left(\zeta_{l k}^{A}\right)$ and coefficients $\left(c_{l k}^{A}\right)$ for the $6-31 \mathrm{G}^{*}$ basis set.

\begin{tabular}{|c|c|c|c|c|c|c|c|c|c|c|c|c|}
\hline Atom & $l$ & $c_{l k}^{A}$ & $\zeta_{l k}^{A}$ & $l$ & $c_{l k}^{A}$ & $\zeta_{l k}^{A}$ & $l$ & $c_{l k}^{A}$ & $\zeta_{l k}^{A}$ & $l$ & $c_{l k}^{A}$ & $\zeta_{l k}^{A}$ \\
\hline \multirow[t]{4}{*}{$\mathrm{H}$} & l & 0.08 & -0.0060132568 & 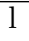 & 0.30 & -0.0737467083 & $\mathrm{~s}$ & 0.08 & -0.0153912737 & $\mathrm{~s}$ & 4.00 & -0.9006446692 \\
\hline & 1 & 0.10 & 0.0389146839 & 1 & 0.60 & 0.1560788345 & $\mathrm{~s}$ & 0.14 & 0.0430589190 & & & \\
\hline & 1 & 0.12 & -0.0601959090 & 1 & 1.00 & -0.2852501671 & $\mathrm{~s}$ & 0.40 & -0.0905475533 & & & \\
\hline & l & 0.18 & 0.0593697573 & 1 & 2.00 & 0.5714955636 & $\mathrm{~s}$ & 1.00 & 0.1541862589 & & & \\
\hline \multirow[t]{3}{*}{ B } & l & 0.08 & 0.0010807452 & 1 & 0.70 & -0.0073045763 & $\mathrm{~S}$ & 0.60 & -0.0134684663 & $\mathrm{~d}$ & 0.08 & -0.0024031037 \\
\hline & 1 & 0.10 & -0.0047823962 & 1 & 0.80 & -0.0031427954 & $\mathrm{p}$ & 0.08 & 0.0471233038 & $\mathrm{a}$ & 0.40 & 0.0634739669 \\
\hline & l & 0.20 & 0.0113519538 & $\mathrm{~S}$ & 0.10 & -0.0286909386 & $\mathrm{p}$ & 0.12 & -0.0668014916 & & & \\
\hline \multirow[t]{5}{*}{$\mathrm{C}$} & l & 0.08 & 0.0004500845 & 1 & 0.80 & 0.0729883176 & $\mathrm{p}$ & 0.08 & 0.0673743263 & $\mathrm{~d}$ & 0.30 & -0.1263858585 \\
\hline & 1 & 0.10 & -0.0020741981 & 1 & 1.80 & -0.1244786722 & $\mathrm{p}$ & 0.14 & -0.0411262590 & $\mathrm{~d}$ & 1.20 & 0.4915351853 \\
\hline & 1 & 0.12 & -0.0012613969 & $\mathrm{~S}$ & 0.10 & -0.0805367963 & $\mathrm{p}$ & 0.16 & -0.0776422890 & & & \\
\hline & 1 & 0.20 & 0.0102034011 & $\mathrm{~S}$ & 0.28 & 0.2101554465 & $\mathrm{~d}$ & 0.08 & -0.0323807736 & & & \\
\hline & 1 & 0.40 & -0.0027191029 & $S$ & 1.20 & -0.4331618971 & $\mathrm{~d}$ & 0.14 & 0.0934454801 & & & \\
\hline \multirow[t]{4}{*}{$\mathrm{N}$} & 1 & 0.08 & 0.0018645260 & l & 0.30 & 0.0152710484 & $\mathrm{~s}$ & 1.40 & 0.1485451212 & 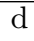 & 0.12 & 0.1309355997 \\
\hline & 1 & 0.12 & -0.0002395552 & $\mathrm{~S}$ & 0.08 & 0.1879576986 & $\mathrm{p}$ & 0.24 & -0.0508315068 & $\mathrm{~d}$ & 0.30 & -0.0789278633 \\
\hline & l & 0.14 & -0.0056636830 & $\mathrm{~s}$ & 0.18 & -0.3622599736 & $\mathrm{p}$ & 0.26 & -0.0032494420 & & & \\
\hline & 1 & 0.28 & 0.0183304638 & $\mathrm{~S}$ & 1.20 & 0.1670603661 & $\mathrm{~d}$ & 0.08 & -0.0885514017 & & & \\
\hline \multirow[t]{4}{*}{$\mathrm{O}$} & 1 & 0.08 & -0.0039627051 & 1 & 0.40 & 0.1330597673 & $\mathrm{~s}$ & 0.28 & -0.6107599468 & $\mathrm{p}$ & 3.00 & 0.5083647602 \\
\hline & 1 & 0.10 & 0.0012915899 & 1 & 0.50 & 0.0092278660 & $\mathrm{p}$ & 0.08 & -0.1938466500 & $\mathrm{~d}$ & 0.08 & 0.1701026799 \\
\hline & 1 & 0.14 & 0.0082667451 & 1 & 2.00 & 0.7543178259 & $\mathrm{p}$ & 0.16 & 0.5522027562 & $\mathrm{~d}$ & 0.14 & -0.3774794563 \\
\hline & 1 & 0.24 & -0.0253267461 & $\mathrm{~S}$ & 0.08 & 0.1103598670 & $\mathrm{p}$ & 0.50 & -1.0606925000 & $\mathrm{~d}$ & 0.50 & 0.2088281812 \\
\hline \multirow[t]{4}{*}{$\bar{F}$} & $\bar{l}$ & 0.08 & 0.0001247392 & 1 & 0.50 & 0.0415363041 & $\mathrm{p}$ & 0.14 & 0.2574015652 & $\mathrm{~d}$ & 0.18 & -0.2261279547 \\
\hline & 1 & 0.14 & -0.0043310646 & $\mathrm{~s}$ & 0.08 & -0.0120194930 & $\mathrm{p}$ & 0.50 & -0.6952103405 & & & \\
\hline & 1 & 0.30 & 0.0375519852 & $\mathrm{~S}$ & 0.90 & -0.2915182575 & $\mathrm{p}$ & 3.00 & 0.9469811796 & & & \\
\hline & 1 & 0.40 & 0.0126943044 & $\mathrm{p}$ & 0.08 & -0.0681355121 & $\mathrm{~d}$ & 0.08 & 0.0470883110 & & & \\
\hline \multirow[t]{3}{*}{$\mathrm{Si}$} & l & 0.08 & 0.0046274494 & l & 0.60 & -0.1725856323 & $\mathrm{p}$ & 0.08 & -0.0003663744 & d & 0.30 & 0.0090963705 \\
\hline & 1 & 0.10 & -0.0103241620 & $\mathrm{~S}$ & 0.08 & 0.0080914989 & $\mathrm{p}$ & 0.28 & 0.0389691222 & $\mathrm{~d}$ & 4.00 & 0.0645634457 \\
\hline & 1 & 0.20 & 0.0191755090 & $\mathrm{~S}$ & 0.10 & 0.0370204050 & $\mathrm{~d}$ & 0.10 & -0.0095545083 & & & \\
\hline \multirow[t]{4}{*}{$\mathrm{P}$} & $\bar{l}$ & 0.08 & 0.0019838068 & $\mathrm{~S}$ & 0.08 & 0.0226354464 & $\mathrm{p}$ & 0.30 & 0.0340568595 & $\mathrm{~d}$ & 0.40 & 0.0314494811 \\
\hline & l & 0.12 & -0.0096021308 & $\mathrm{~S}$ & 0.10 & 0.0038366730 & $\mathrm{p}$ & 0.40 & 0.0084848656 & & & \\
\hline & 1 & 0.22 & 0.0384446317 & $\mathrm{~s}$ & 0.26 & 0.0130261307 & $\mathrm{~d}$ & 0.08 & -0.0048673060 & & & \\
\hline & 1 & 0.40 & -0.1266731084 & $\mathrm{p}$ & 0.08 & -0.0064576993 & $\mathrm{~d}$ & 0.30 & 0.0139040096 & & & \\
\hline \multirow[t]{3}{*}{$\mathrm{S}$} & 1 & 0.08 & -0.0014007289 & 1 & 0.26 & 0.0563257153 & $\mathrm{~S}$ & 0.40 & 0.1429486274 & $\mathrm{~d}$ & 0.08 & 0.0253267431 \\
\hline & 1 & 0.10 & 0.0026697233 & 1 & 0.50 & -0.2120846053 & $\mathrm{p}$ & 0.10 & -0.0045772361 & $\mathrm{~d}$ & 0.22 & -0.0550823688 \\
\hline & l & 0.14 & -0.0120623483 & $\mathrm{~s}$ & 0.08 & -0.0192279111 & $\mathrm{p}$ & 0.60 & 0.1413103298 & & & \\
\hline \multirow[t]{3}{*}{$\mathrm{Cl}$} & 1 & 0.08 & -0.0045464436 & 1 & 0.30 & 0.0318108048 & $\mathrm{~S}$ & 0.08 & 0.0650152336 & $p$ & 0.14 & -0.0179496435 \\
\hline & l & 0.10 & 0.0127959301 & 1 & 0.70 & -0.0364908596 & $\mathrm{~s}$ & 4.00 & 0.1092818169 & $\mathrm{~d}$ & 0.12 & -0.0732051934 \\
\hline & l & 0.16 & -0.0089215218 & 1 & 0.80 & -0.0802160134 & $\mathrm{p}$ & 0.08 & 0.0057024006 & & & \\
\hline
\end{tabular}


TABLE XIV. BSIP exponents $\left(\zeta_{l k}^{A}\right)$ and coefficients $\left(c_{l k}^{A}\right)$ for the Def2-SV $(\mathrm{P})$ basis set.

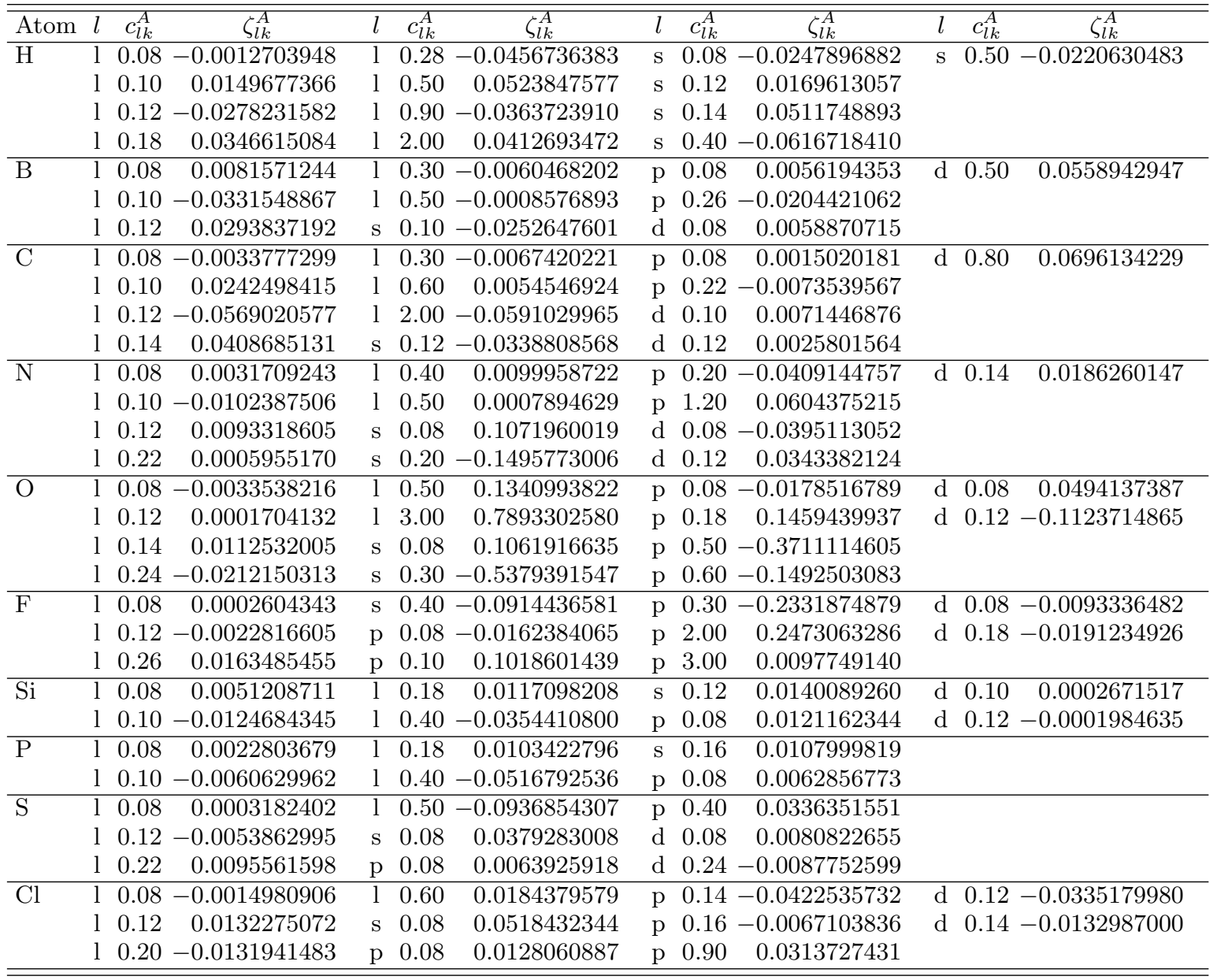


TABLE XV. BSIP exponents $\left(\zeta_{l k}^{A}\right)$ and coefficients $\left(c_{l k}^{A}\right)$ for the Def2-SVP basis set.

\begin{tabular}{|c|c|c|c|c|c|c|c|c|c|c|c|}
\hline Atom & $l$ & $c_{l k}^{A}$ & $\zeta_{l k}^{A}$ & $l$ & $c_{l k}^{A}$ & $\zeta_{l k}^{A}$ & $l$ & $c_{l k}^{A}$ & $\zeta_{l k}^{A}$ & $l \quad c_{l k}^{A}$ & $\zeta_{l k}^{A}$ \\
\hline \multirow[t]{4}{*}{$\mathrm{H}$} & l & 0.08 & 0.0010488991 & 1 & 0.30 & -0.0390576134 & $\mathrm{~s}$ & 0.08 & 0.0101015178 & $\mathrm{p} \quad 0.40$ & -0.0644417847 \\
\hline & 1 & 0.10 & 0.0045185898 & 1 & 0.50 & 0.0492450115 & $\mathrm{~s}$ & 0.30 & -0.0157524854 & p 1.20 & 0.2714729043 \\
\hline & l & 0.12 & -0.0150103520 & l & 0.60 & 0.0013900452 & $\mathrm{p}$ & 0.08 & -0.0182108995 & p 1.40 & 0.0393930928 \\
\hline & 1 & 0.18 & 0.0254001217 & 1 & 1.20 & -0.0657632443 & $\mathrm{p}$ & 0.12 & 0.0269266702 & & \\
\hline \multirow[t]{3}{*}{ B } & l & 0.08 & 0.0003667643 & 1 & 0.24 & 0.0050504025 & $\mathrm{p}$ & 0.22 & -0.0283454804 & & \\
\hline & 1 & 0.10 & -0.0021958133 & $\mathrm{~s}$ & 0.14 & -0.0333108482 & $\mathrm{~d}$ & 0.08 & 0.0033620243 & & \\
\hline & 1 & 0.22 & 0.0020119948 & $\mathrm{p}$ & 0.08 & 0.0079126415 & d & 0.30 & 0.0351184474 & & \\
\hline \multirow[t]{4}{*}{$\mathrm{C}$} & l & 0.08 & -0.0007838636 & 1 & 0.60 & 0.0367484391 & $\mathrm{p}$ & 0.08 & 0.0227505403 & d 0.18 & 0.0098441476 \\
\hline & 1 & 0.10 & -0.0002933940 & 1 & 2.00 & -0.1756719913 & $\mathrm{p}$ & 0.16 & -0.0053346241 & & \\
\hline & 1 & 0.16 & 0.0025785416 & $\mathrm{~S}$ & 0.10 & -0.0342829665 & $\mathrm{p}$ & 0.18 & -0.0413489301 & & \\
\hline & l & 0.18 & 0.0009974840 & $\mathrm{~S}$ & 0.90 & -0.0021487389 & $\mathrm{~d}$ & 0.08 & -0.0008446953 & & \\
\hline \multirow[t]{3}{*}{$\mathrm{N}$} & 1 & 0.08 & 0.0009293386 & 1 & 0.40 & 0.0067970476 & $\mathrm{~s}$ & 0.20 & -0.1556569854 & d 0.08 & -0.0406130604 \\
\hline & 1 & 0.10 & -0.0009191487 & 1 & 0.50 & 0.0097842263 & $\mathrm{p}$ & 0.08 & 0.0152842442 & d 0.14 & 0.0545140699 \\
\hline & 1 & 0.30 & 0.0094295960 & $\mathrm{~s}$ & 0.08 & 0.0961292626 & $\mathrm{p}$ & 0.20 & -0.0635918701 & & \\
\hline \multirow[t]{4}{*}{$\mathrm{O}$} & 1 & 0.08 & -0.0036359101 & 1 & 0.50 & 0.0725879893 & $\mathrm{p}$ & 0.16 & 0.0933266554 & d 0.14 & -0.1435177664 \\
\hline & l & 0.10 & 0.0009709378 & 1 & 3.00 & 0.7651151212 & $\mathrm{p}$ & 0.50 & -0.1111249373 & d 0.50 & 0.0770430274 \\
\hline & l & 0.14 & 0.0106197946 & $\mathrm{~s}$ & 0.08 & 0.0883269765 & $\mathrm{p}$ & 0.60 & -0.3596844442 & d 0.60 & 0.2676580926 \\
\hline & l & 0.16 & -0.0062512089 & $\mathrm{~S}$ & 0.28 & -0.4817963932 & $\mathrm{~d}$ & 0.08 & 0.0366053731 & & \\
\hline \multirow[t]{3}{*}{$\mathrm{F}$} & ] & 0.08 & 0.0002956992 & 1 & 0.24 & 0.0176525868 & $\mathrm{p}$ & 0.08 & 0.0145027541 & $\mathrm{p} 2.00$ & 0.1023560112 \\
\hline & l & 0.10 & -0.0001584670 & $\mathrm{~s}$ & 0.08 & -0.0110673220 & $\mathrm{p}$ & 0.10 & 0.0438438961 & d 0.08 & -0.0033732861 \\
\hline & l & 0.12 & -0.0026023248 & $\mathrm{~s}$ & 0.28 & -0.0427914938 & $\mathrm{p}$ & 0.30 & -0.1725777429 & d 0.18 & -0.0319216611 \\
\hline \multirow[t]{2}{*}{$\mathrm{Si}$} & l & 0.08 & -0.0000488966 & 1 & 0.16 & 0.0073010882 & $\mathrm{~s}$ & 0.08 & 0.0085370514 & $\mathrm{p} \quad 0.10$ & -0.0777496439 \\
\hline & 1 & 0.10 & -0.0027392173 & 1 & 0.40 & -0.0240440438 & $\mathrm{p}$ & 0.08 & 0.0692457912 & d 0.10 & -0.0049648383 \\
\hline \multirow[t]{2}{*}{$\mathrm{P}$} & l & 0.08 & 0.0028514105 & 1 & 0.18 & 0.0090094128 & l & 0.50 & -0.0629180439 & $\mathrm{p} \quad 0.30$ & -0.0068832829 \\
\hline & 1 & 0.10 & -0.0060555158 & 1 & 0.20 & 0.0016885511 & $\mathrm{~s}$ & 0.14 & 0.0102349225 & d 0.08 & -0.0022620309 \\
\hline \multirow[t]{2}{*}{$\mathrm{S}$} & 1 & 0.08 & 0.0009911951 & l & 0.22 & 0.0030159226 & $\mathrm{~s}$ & 0.08 & 0.0207620421 & d 0.08 & 0.0137406360 \\
\hline & 1 & 0.10 & -0.0031221587 & 1 & 0.50 & -0.0466352106 & $\mathrm{p}$ & 0.08 & 0.0033631442 & d 0.20 & -0.0301124733 \\
\hline \multirow[t]{3}{*}{$\mathrm{Cl}$} & 1 & 0.08 & -0.0015597220 & 1 & 0.50 & 0.0213531272 & $\mathrm{p}$ & 0.16 & -0.0723257712 & & \\
\hline & 1 & 0.12 & 0.0134497278 & $\mathrm{~s}$ & 0.08 & 0.0342944666 & d & 0.12 & -0.0195429125 & & \\
\hline & l & 0.20 & -0.0113722155 & $\mathrm{p}$ & 0.08 & 0.0211415043 & $\mathrm{~d}$ & 0.14 & -0.0354835840 & & \\
\hline
\end{tabular}


TABLE XVI. BSIP exponents $\left(\zeta_{l k}^{A}\right)$ and coefficients $\left(c_{l k}^{A}\right)$ for the pc-1 basis set.

\begin{tabular}{|c|c|c|c|c|c|c|c|c|c|c|c|c|}
\hline Atom & $l$ & $c_{l k}^{A}$ & $\zeta_{l k}^{A}$ & $l$ & $c_{l k}^{A}$ & $\zeta_{l k}^{A}$ & $l$ & $c_{l k}^{A}$ & $\zeta_{l k}^{A}$ & $l$ & $c_{l k}^{A}$ & $\zeta_{l k}^{A}$ \\
\hline \multirow[t]{4}{*}{$\mathrm{H}$} & & 0.08 & -0.0018588046 & 1 & 0.26 & 1.1326735381 & $\mathrm{~s}$ & 0.08 & 0.0200778151 & $\mathrm{p}$ & 0.08 & -0.0119866363 \\
\hline & 1 & 0.10 & 0.0126490629 & 1 & 0.28 & -2.3323305283 & $\mathrm{~s}$ & 0.14 & -0.0357279374 & $\mathrm{p}$ & 0.18 & 0.0467246957 \\
\hline & l & 0.12 & -0.0105851088 & 1 & 0.30 & 1.2309253828 & $\mathrm{~s}$ & 0.90 & 0.0825758968 & $\mathrm{p}$ & 0.40 & -0.0938812315 \\
\hline & l & 0.14 & -0.0167607180 & 1 & 0.80 & -0.0278139827 & $\mathrm{~s}$ & 4.00 & -0.2436838676 & $\mathrm{p}$ & 1.20 & 0.2627387519 \\
\hline \multirow[t]{3}{*}{ B } & 1 & 0.08 & -0.0017500114 & 1 & 0.40 & 0.0061245389 & $\mathrm{~s}$ & 0.60 & -0.0342521758 & $\mathrm{~d}$ & 0.08 & 0.0158285188 \\
\hline & 1 & 0.12 & 0.0096243557 & 1 & 1.20 & -0.0180781626 & $\mathrm{p}$ & 0.08 & 0.0021834518 & $\mathrm{~d}$ & 0.12 & -0.0317506020 \\
\hline & 1 & 0.14 & -0.0093615717 & $\mathrm{~S}$ & 0.10 & -0.0025406389 & $\mathrm{p}$ & 0.14 & -0.0061847563 & $\mathrm{~d}$ & 0.40 & 0.0703391171 \\
\hline \multirow[t]{6}{*}{$\mathrm{C}$} & l & 0.08 & -0.0015268922 & 1 & 2.00 & -0.5939296413 & $\mathrm{p}$ & 0.14 & -0.1034370373 & $\mathrm{~d}$ & 0.30 & -0.2119260074 \\
\hline & 1 & 0.10 & 0.0073955195 & $\mathrm{~s}$ & 0.08 & 0.0026277491 & $\mathrm{p}$ & 0.16 & -0.0182127172 & $\mathrm{~d}$ & 1.00 & 0.1484433415 \\
\hline & 1 & 0.14 & -0.0200548921 & $\mathrm{~s}$ & 0.12 & -0.0930528706 & $\mathrm{p}$ & 0.70 & 0.1748711168 & $\mathrm{~d}$ & 1.20 & 0.2473505502 \\
\hline & 1 & 0.22 & 0.0339537506 & $\mathrm{~s}$ & 0.26 & 0.1739312542 & d & 0.08 & -0.0452516539 & & & \\
\hline & 1 & 0.40 & -0.0642761321 & $\mathrm{~s}$ & 1.00 & -0.0470923991 & d & 0.14 & 0.0758131410 & & & \\
\hline & l & 0.70 & 0.1358806107 & $\mathrm{p}$ & 0.08 & 0.0666655686 & $\mathrm{~d}$ & 0.16 & 0.0910027777 & & & \\
\hline \multirow[t]{4}{*}{$\mathrm{N}$} & $\bar{l}$ & 0.08 & 0.0039929263 & l & 0.40 & 0.0201978666 & $\mathrm{p}$ & 0.10 & 0.0326716469 & $\mathrm{~d}$ & 0.08 & -0.0682270969 \\
\hline & 1 & 0.10 & -0.0089160611 & $\mathrm{~s}$ & 0.08 & 0.0970015864 & $\mathrm{p}$ & 0.22 & -0.0912357239 & $\mathrm{~d}$ & 0.12 & 0.0997297620 \\
\hline & 1 & 0.16 & 0.0332182293 & $\mathrm{~S}$ & 0.18 & -0.1763551690 & $\mathrm{p}$ & 0.70 & 0.0249556433 & d & 0.26 & -0.0283271619 \\
\hline & l & 0.18 & -0.0323338299 & $\mathrm{~S}$ & 0.70 & 0.0633770042 & $\mathrm{p}$ & 0.80 & 0.0193975447 & & & \\
\hline \multirow[t]{4}{*}{$\mathrm{O}$} & l & 0.08 & -0.0023582597 & 1 & 0.50 & 0.0297249314 & $\mathrm{p}$ & 0.08 & -0.0982348537 & $\mathrm{~d}$ & 0.08 & 0.0947203933 \\
\hline & l & 0.10 & 0.0004471492 & l & 2.00 & 0.7568447231 & $\mathrm{p}$ & 0.16 & 0.2605607710 & $\mathrm{~d}$ & 0.14 & -0.2031006798 \\
\hline & 1 & 0.16 & 0.0032507182 & $\mathrm{~S}$ & 0.10 & 0.1502405778 & $\mathrm{p}$ & 0.50 & -0.1632008043 & 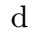 & 0.50 & 0.0730032501 \\
\hline & l & 0.40 & 0.0183650653 & $\mathrm{~s}$ & 0.26 & -0.5599186035 & $\mathrm{p}$ & 0.60 & -0.4317172984 & $\mathrm{~d}$ & 0.60 & 0.1131195456 \\
\hline \multirow[t]{4}{*}{$\mathrm{F}$} & l & 0.08 & 0.0008608940 & 1 & 0.40 & -0.0056667496 & $\mathrm{p}$ & 0.14 & 0.1951938898 & $\mathrm{~d}$ & 0.08 & 0.0498689163 \\
\hline & l & 0.12 & -0.0062788594 & $\mathrm{~S}$ & 0.14 & 0.0196311300 & $\mathrm{p}$ & 0.40 & -0.1209826838 & 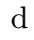 & 0.16 & -0.1319100206 \\
\hline & 1 & 0.20 & 0.0103413960 & $\mathrm{~s}$ & 0.60 & -0.3275614720 & $\mathrm{p}$ & 0.50 & -0.2393387676 & d & 0.50 & 0.0955011099 \\
\hline & 1 & 0.22 & 0.0063244891 & $\mathrm{p}$ & 0.08 & -0.0739955766 & $\mathrm{p}$ & 3.00 & 0.5459302816 & & & \\
\hline \multirow[t]{3}{*}{$\mathrm{Si}$} & & 0.08 & 0.0002532828 & 1 & 0.24 & 0.0179835581 & $\mathrm{~s}$ & 0.08 & -0.0019383439 & $\mathrm{p}$ & 0.20 & 0.0368093448 \\
\hline & & 0.12 & 0.0019069449 & 1 & 0.60 & -0.0442095426 & $\mathrm{~s}$ & 0.20 & 0.0885792608 & d & 0.08 & 0.003061 \\
\hline & 1 & 0.14 & -0.0114388740 & 1 & 0.70 & -0.1056828547 & $\mathrm{p}$ & 0.08 & 0.0018279583 & d & 0.16 & -0.0040183857 \\
\hline \multirow[t]{3}{*}{$\mathrm{P}$} & 1 & 0.08 & 0.0017616038 & 1 & 0.40 & -0.0686877430 & $\mathrm{p}$ & 0.30 & 0.0122818489 & & & \\
\hline & ] & 0.12 & -0.0082972783 & $\mathrm{~S}$ & 0.24 & 0.0132413986 & $\mathrm{~d}$ & 0.08 & -0.0009517266 & & & \\
\hline & 1 & 0.22 & 0.0225241489 & $\mathrm{p}$ & 0.28 & 0.0065935012 & $\mathrm{~d}$ & 0.24 & 0.0151651368 & & & \\
\hline \multirow[t]{3}{*}{$\mathrm{S}$} & l & 0.08 & -0.0019854409 & 1 & 0.26 & 0.0368635872 & $\mathrm{p}$ & 0.08 & -0.0050498766 & $\mathrm{~d}$ & 0.28 & -0.0511583338 \\
\hline & 1 & 0.10 & 0.0045197220 & 1 & 0.50 & -0.1188180788 & $\mathrm{p}$ & 0.50 & 0.0876857945 & & & \\
\hline & l & 0.16 & -0.0161566268 & $\mathrm{~S}$ & 0.26 & 0.0502230991 & $\mathrm{~d}$ & 0.08 & 0.0164835126 & & & \\
\hline \multirow[t]{3}{*}{$\mathrm{Cl}$} & l & 0.08 & -0.0015156649 & 1 & 0.20 & -0.0124041986 & $\mathrm{~s}$ & 0.22 & 0.0608422484 & 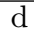 & 0.16 & -0.0225196678 \\
\hline & l & 0.10 & 0.0054411595 & 1 & 0.22 & -0.0014369881 & $\mathrm{p}$ & 0.12 & -0.0155263812 & d & 0.18 & -0.0487739763 \\
\hline & l & 0.14 & 0.0054351940 & 1 & 0.40 & 0.0241025357 & $\mathrm{p}$ & 0.50 & 0.0046325217 & & & \\
\hline
\end{tabular}


TABLE XVII. BSIP exponents $\left(\zeta_{l k}^{A}\right)$ and coefficients $\left(c_{l k}^{A}\right)$ for the $6-31+\mathrm{G}^{*}$ basis set.

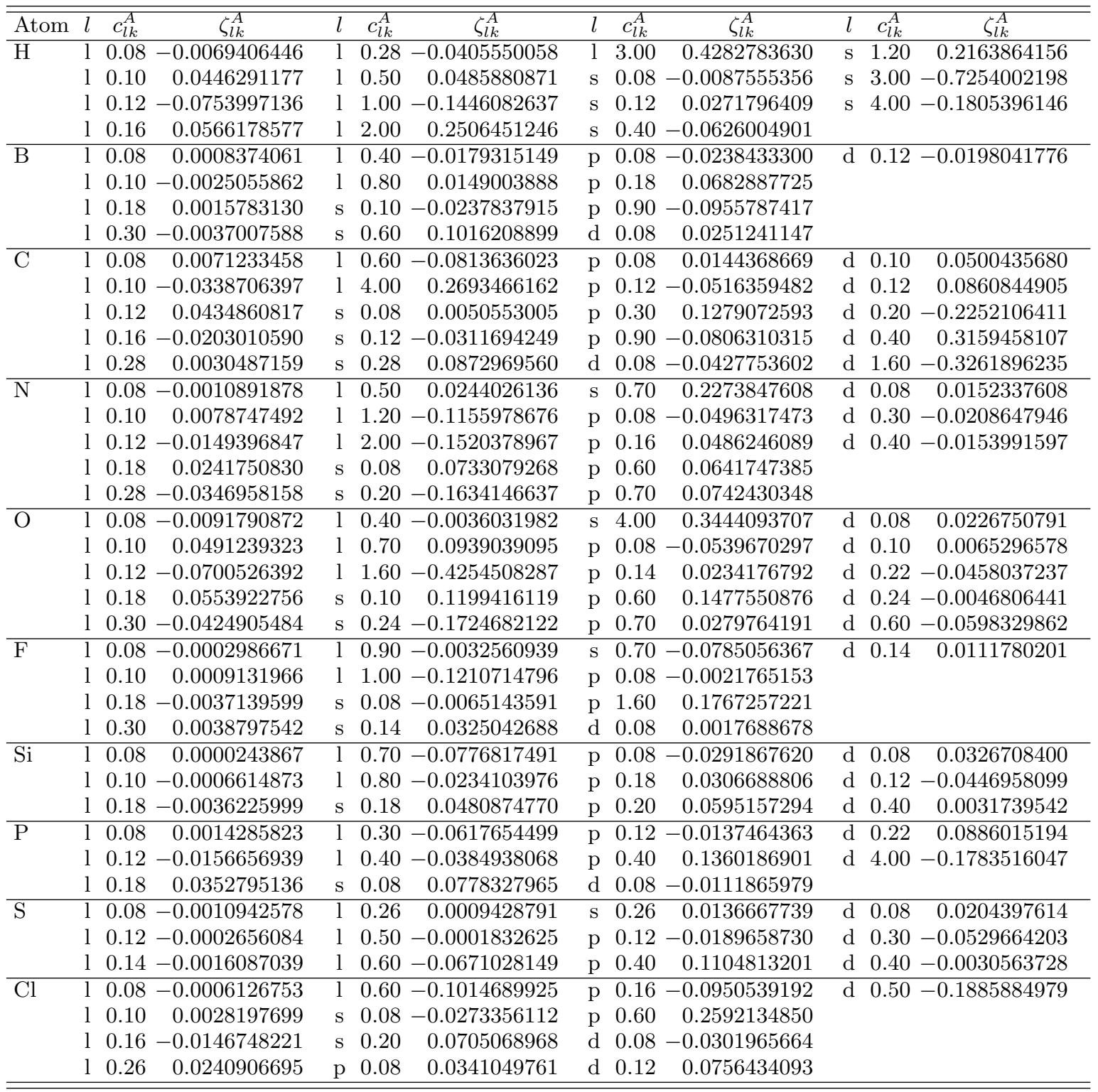


TABLE XVIII. BSIP exponents $\left(\zeta_{l k}^{A}\right)$ and coefficients $\left(c_{l k}^{A}\right)$ for the $6-31+\mathrm{G}^{* *}$ basis set.

\begin{tabular}{|c|c|c|c|c|c|c|c|c|c|c|c|c|}
\hline Atom & $l$ & $\overline{c_{l k}^{A}}$ & $\overline{\zeta_{l k}^{A}}$ & $l$ & $\overline{c_{l k}^{A}}$ & $\overline{\zeta_{l k}^{A}}$ & $l$ & $\overline{c_{l k}^{A}}$ & $\overline{\zeta_{l k}^{A}}$ & & $c_{l k}^{A}$ & $\zeta_{l k}^{A}$ \\
\hline \multirow[t]{5}{*}{$\mathrm{H}$} & l & 0.08 & -0.0036992299 & l & 0.26 & -0.0203785804 & l & 4.00 & -0.4805303961 & $\mathrm{p}$ & 0.08 & -0.0143782167 \\
\hline & 1 & 0.10 & 0.0282915096 & 1 & 0.40 & 0.0394978716 & $\mathrm{~s}$ & 0.08 & 0.0064312472 & $\mathrm{p}$ & 0.10 & 0.0227818795 \\
\hline & l & $0.1 \%$ & -0.0540427020 & l & 0.80 & -0.0856720882 & $\mathrm{~s}$ & 0.16 & -0.0044197545 & $\mathrm{p}$ & 0.16 & -0.0099145796 \\
\hline & l & 0.16 & 0.0515314623 & l & 1.40 & 0.1604351762 & $\mathrm{~s}$ & 0.40 & -0.0225905456 & $\mathrm{p}$ & 0.30 & -0.0114485976 \\
\hline & 1 & 0.2 & -0.0232632122 & 1 & 3.00 & -0.2944475760 & $\mathrm{~s}$ & 1.80 & 0.3660703189 & $\mathrm{p}$ & 0.70 & 0.0851614377 \\
\hline \multirow[t]{4}{*}{ B } & 1 & 0.08 & -0.0002713820 & 1 & 0.40 & -0.0157212834 & $\mathrm{p}$ & 0.18 & 0.0495393557 & $\mathrm{~d}$ & 0.24 & 0.0025049528 \\
\hline & l & 0.10 & 0.0010519580 & $\mathrm{~s}$ & 0.08 & -0.0144861755 & $\mathrm{p}$ & 0.70 & -0.0521566354 & & & \\
\hline & l & 0.12 & -0.0038581732 & $\mathrm{~s}$ & 0.60 & 0.0805477411 & $\mathrm{~d}$ & 0.08 & 0.0236196972 & & & \\
\hline & 1 & 0.2 & 0.0024884491 & $\mathrm{p}$ & 0.08 & -0.0159677651 & d & 0.10 & -0.0170929152 & & & \\
\hline \multirow[t]{6}{*}{$\mathrm{C}$} & 1 & 0.08 & 0.0060412220 & l & 0.60 & -0.0481368129 & $\mathrm{~s}$ & 0.90 & -0.0095117962 & $\mathrm{~d}$ & 0.10 & 0.1092953861 \\
\hline & 1 & 0.10 & -0.0305390703 & 1 & 4.00 & 0.4876392348 & $\mathrm{p}$ & 0.08 & 0.0187395097 & d & 0.18 & -0.0895882653 \\
\hline & l & $0.1 \%$ & 0.0435574622 & $\mathrm{~s}$ & 0.08 & 0.0073404663 & $\mathrm{p}$ & 0.14 & -0.0594121267 & $\mathrm{~d}$ & 0.40 & 0.1069777000 \\
\hline & 1 & 0.16 & -0.0274584400 & $\mathrm{~s}$ & 0.12 & -0.0520762795 & $\mathrm{p}$ & 0.30 & 0.1158899027 & d & 2.00 & -0.2486727399 \\
\hline & 1 & 0.26 & 0.0100231022 & $\mathrm{~s}$ & 0.28 & 0.1728275677 & $\mathrm{p}$ & 0.90 & -0.1452207184 & & & \\
\hline & 1 & 0.50 & -0.0010863015 & $\mathrm{~s}$ & 0.80 & -0.1882141481 & d & 0.08 & -0.0582476565 & & & \\
\hline \multirow[t]{5}{*}{$\mathrm{N}$} & 1 & 0.08 & -0.0002352113 & l & 0.50 & 0.0535907194 & $\mathrm{~s}$ & 0.20 & -0.1524523444 & $\mathrm{p}$ & 0.80 & 0.1959512874 \\
\hline & 1 & 0.10 & 0.0069218855 & 1 & 1.00 & -0.0623224916 & $\mathrm{~s}$ & 0.70 & 0.2345244504 & d & 0.08 & 0.0115460601 \\
\hline & 1 & $0.1 \%$ & -0.0159288198 & l & 1.20 & -0.1475190573 & $\mathrm{p}$ & 0.08 & -0.0543578568 & $\mathrm{~d}$ & 0.30 & -0.0093936918 \\
\hline & 1 & 0.18 & 0.0255327108 & 1 & 3.00 & -0.1429370889 & $\mathrm{p}$ & 0.14 & 0.0753083067 & d & 0.40 & -0.0264164356 \\
\hline & 1 & $0.2 \varepsilon$ & -0.0391795989 & $\mathrm{~s}$ & 0.08 & 0.0663257925 & $\mathrm{p}$ & 0.30 & -0.0447610051 & & & \\
\hline \multirow[t]{5}{*}{$\mathrm{O}$} & 1 & 0.08 & -0.0080620035 & l & 0.60 & 0.0608131335 & $\mathrm{~s}$ & 3.00 & 0.6142954670 & $\mathrm{p}$ & 3.00 & -0.2607483490 \\
\hline & 1 & 0.10 & 0.0448187691 & 1 & 1.40 & -0.1307378174 & $\mathrm{p}$ & 0.08 & -0.0333164541 & d & 0.08 & 0.0144510923 \\
\hline & 1 & 0.12 & -0.0649894596 & $\mathrm{~s}$ & 0.08 & -0.0008890547 & $\mathrm{p}$ & 0.16 & 0.0015545913 & d & 0.16 & -0.0084010377 \\
\hline & l & 0.18 & 0.0538224132 & $\mathrm{~s}$ & 0.12 & 0.1658352506 & $\mathrm{p}$ & 0.18 & 0.0009532497 & $\mathrm{~d}$ & 1.00 & -0.2444681000 \\
\hline & 1 & 0.30 & -0.0483358073 & $\mathrm{~s}$ & 0.28 & -0.3955362372 & $\mathrm{p}$ & 0.60 & 0.1505198465 & & & \\
\hline \multirow[t]{4}{*}{$\mathrm{F}$} & 1 & 0.08 & 0.0000382628 & 1 & 0.60 & -0.0413978741 & $\mathrm{p}$ & 0.14 & 0.0254067958 & $\mathrm{~d}$ & 0.14 & 0.0081148948 \\
\hline & 1 & 0.10 & 0.0003168789 & $\mathrm{~s}$ & 0.08 & -0.0093677117 & $\mathrm{p}$ & 0.30 & -0.0465231021 & & & \\
\hline & 1 & 0.18 & -0.0043372543 & $\mathrm{~s}$ & 0.16 & 0.0046786062 & $\mathrm{p}$ & 1.40 & 0.1285336936 & & & \\
\hline & 1 & 0.30 & 0.0103001109 & $\mathrm{p}$ & 0.08 & -0.0030863137 & $\mathrm{~d}$ & 0.12 & 0.0021197662 & & & \\
\hline \multirow[t]{4}{*}{$\mathrm{Si}$} & 1 & 0.08 & -0.0000130810 & l & 0.40 & -0.0103929969 & $\mathrm{p}$ & 0.08 & -0.0139903884 & $\mathrm{~d}$ & 0.40 & 0.0016638831 \\
\hline & 1 & 0.10 & -0.0015448651 & 1 & 0.80 & -0.0681134622 & $\mathrm{p}$ & 0.24 & 0.0677635553 & & & \\
\hline & 1 & 0.14 & 0.0021181767 & $\mathrm{~s}$ & 0.08 & -0.0128677618 & d & 0.08 & 0.0246456833 & & & \\
\hline & 1 & 0.30 & -0.0081450753 & $\mathrm{~s}$ & 0.16 & 0.0601113183 & d & 0.12 & -0.0342179731 & & & \\
\hline \multirow[t]{3}{*}{$\mathrm{P}$} & 1 & 0.08 & 0.0010166290 & l & 0.30 & -0.0214469990 & $\mathrm{~S}$ & 4.00 & -0.0548029934 & $\mathrm{~d}$ & 0.08 & -0.0089779433 \\
\hline & 1 & 0.12 & -0.0112688118 & 1 & 0.40 & -0.0698200410 & $\mathrm{p}$ & 0.12 & -0.0169030537 & d & 0.22 & 0.0718660148 \\
\hline & l & 0.18 & 0.0221440042 & $\mathrm{~s}$ & 0.08 & 0.0714599465 & $\mathrm{p}$ & 0.40 & 0.1335088826 & d & 4.00 & -0.0293463373 \\
\hline \multirow[t]{4}{*}{$\mathrm{S}$} & 1 & 0.08 & -0.0008584488 & 1 & 0.50 & -0.0323843518 & $\mathrm{p}$ & 0.14 & -0.0170153178 & $\mathrm{~d}$ & 0.60 & -0.0012773160 \\
\hline & & 0.10 & 0.0002778689 & 1 & 0.60 & -0.0434713230 & $\mathrm{p}$ & 0.50 & 0.1203795223 & & & \\
\hline & 1 & 0.16 & -0.0047087385 & $\mathrm{~s}$ & 0.08 & 0.0039696493 & d & 0.08 & 0.0118024258 & & & \\
\hline & 1 & 0.28 & 0.0091836363 & $\mathrm{p}$ & 0.08 & 0.0031898149 & d & 0.50 & -0.0533931870 & & & \\
\hline \multirow[t]{4}{*}{$\mathrm{Cl}$} & l & 0.08 & -0.0000375526 & 1 & 0.50 & -0.0165230578 & $\mathrm{~S}$ & 0.22 & 0.1000478734 & d & 0.08 & -0.0152165819 \\
\hline & & $0.1 \%$ & 0.0060479654 & l & 0.60 & -0.1273793434 & $\mathrm{p}$ & 0.08 & 0.0202439425 & $\mathrm{~d}$ & 0.12 & 0.0482743521 \\
\hline & 1 & 0.16 & -0.0203652942 & $\mathrm{~s}$ & 0.08 & -0.0416150349 & $\mathrm{p}$ & 0.16 & -0.0709480218 & d & 0.50 & -0.0789156944 \\
\hline & l & $0.2 \varsigma$ & 0.0328761222 & $\mathrm{~s}$ & 0.20 & 0.0383382290 & $\mathrm{p}$ & 0.50 & 0.2323016452 & $\mathrm{~d}$ & 0.60 & -0.0536311705 \\
\hline
\end{tabular}

